\title{
HOCHSCHILD COHOMOLOGY RING OF THE MODULAR GROUP
}

\author{
A. P. ALEKHIN, YU. V. VOLKOV, AND A. I. GENERALOV
}

\begin{abstract}
A description in terms of generators and relations is given for the cohomology ring and the Hochschild cohomology ring of the group algebra for the even modular group over the ring of integers. The free resolution of the trivial module described by Wall is used for that. Moreover, the bimodule resolution of the group algebra in question is described.
\end{abstract}

\section{§1. INTRODUCTION}

Through the past years, the interest has grown to the investigation of the structure of the Hochschild cohomology algebra. There are many results on calculation of that algebra for finite-dimensional algebras over a field. Nevertheless, not many results are known for group algebras over the ring of integers.

In the papers 1] and 2, the Hochschild cohomology algebra was calculated for the group ring $\mathbb{Z}\left[D_{4 m}\right]$ and its additive structure was described for $\mathbb{Z}\left[S D_{2^{k}}\right]$. In those papers, the bimodule resolution of the corresponding algebra was used as the main tool.

In his papers [3, 4, 5, 6, Hayami described the Hochschild cohomology ring for integer group rings of generalized quaternion groups, of dihedral groups, and of semidihedral groups. He used the results of [7] in his calculations.

The main result of the present paper is the description of the Hochschild cohomology algebra of the integer group ring of the even modular group $M_{2^{\ell}}$. The key construction is the free resolution described by Wall in [8]. Using this resolution and results of [9], we immediately obtain the bimodule resolution of the group algebra in question. Nonetheless, a technique similar to that of [7] allows us to use only the free resolution of the trivial module $\mathbb{Z}$ in the calculation of the Hochschild cohomology algebra.

It should be noted that the results of calculation of the algebra $\mathrm{HH}^{*}\left(\mathbb{Z}\left[M_{2^{\ell}}\right]\right)$ show that this algebra is commutative.

From calculations presented in the paper, we also derive a description of the usual cohomology algebra of the even modular group. Though the cohomology algebra for the modular groups of odd order was calculated in the works [10] by Leary and 11] by Thomas, the authors were not able to find a publication containing a description of the cohomology algebra of the modular groups of even order.

\section{$\S 2$. Free Resolution}

In this paper, we consider only right modules. Moreover, we write simply $\otimes$ instead of $\otimes_{\mathbb{Z}}$. Fixing a natural number $\ell \geq 4$, we introduce the notation

$$
r=2^{\ell-2}, \quad t=r+1 .
$$

2010 Mathematics Subject Classification. Primary 16E40.

Key words and phrases. Hochschild cohomology, group algebras, modular 2-groups. 
The modular group of order $4 r$ can be presented as a semidirect product of two cyclic groups:

$$
G=M_{2^{\ell}}=C_{2} \curlywedge C_{2 r}=\left\langle a, b \mid a^{2}=b^{2 r}=1, a b a=b^{t}\right\rangle .
$$

We consider an integer group algebra $R=\mathbb{Z}[G]$ of the group $G$.

Construction of the resolution is based on the results presented in 8 and employs the following notation:

$$
N_{b}=\sum_{i=0}^{2 r-1} b^{i}, \quad L=\sum_{i=0}^{r} b^{i}, \quad x_{k}=\frac{t^{k}+1}{2}, \quad y_{k}=\frac{t^{k}-1}{r} .
$$

We build the following diagram:

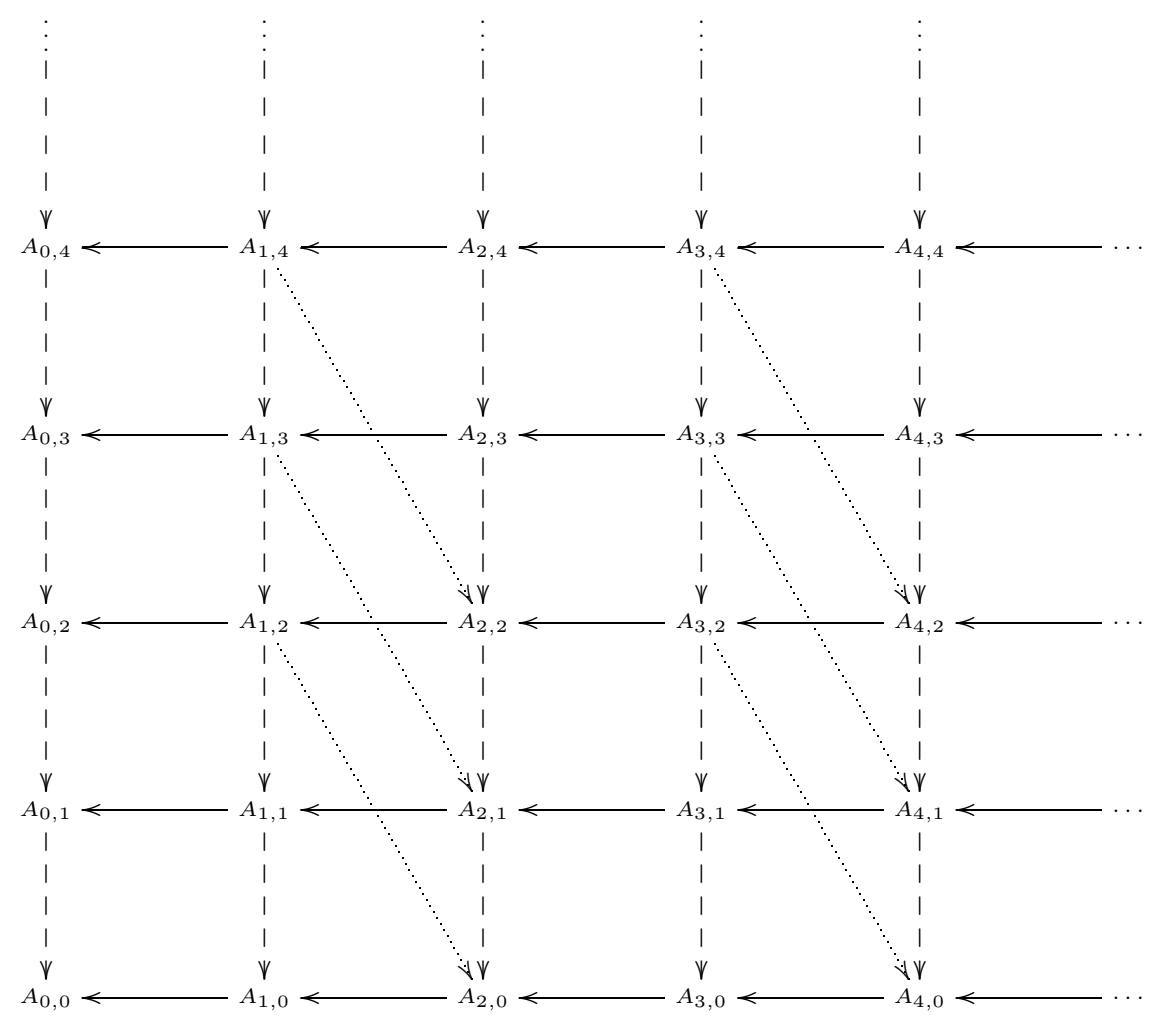

Here, any vertex $A_{i, j}$ is simply the group algebra $R$. The additional numbering is needed for defining the arrows, namely, each arrow represents multiplication (from the left) by the corresponding element of $R$ :

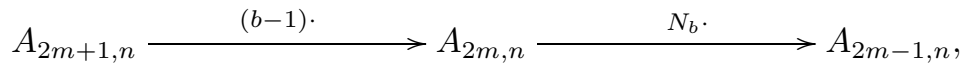

$$
\begin{aligned}
& A_{2 m, 2 n+1}--\stackrel{\left(L^{m} a-1\right)}{-}-->A_{2 m, 2 n}--\stackrel{\left(L^{m} a+1\right)}{-}-\stackrel{-}{-}-A_{2 m, 2 n-1}, \\
& A_{2 m-1,2 n+1}-\stackrel{\left(-L^{m} a+1\right)}{-}-\stackrel{-}{-} \rightarrow A_{2 m-1,2 n}-\stackrel{\left(-L^{m} a-1\right) .}{-}-\stackrel{-}{\rightarrow} A_{2 m-1,2 n-1},
\end{aligned}
$$

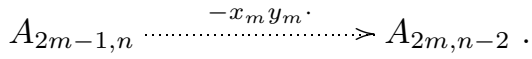


By a process similar to the construction of a total complex of a bicomplex, we use the above diagram to construct the following complex:

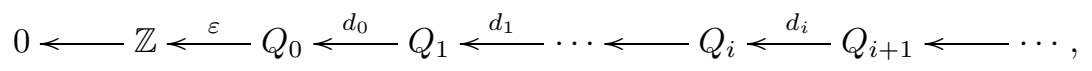

where $Q_{i}=R^{i+1}, \varepsilon$ is the augmentation map $(\varepsilon(1 \cdot g)=1$ for any $g \in G)$, and the differentials are represented by three-diagonal matrices of the following form:

$$
\begin{aligned}
& d_{0}=(a-1, b-1),
\end{aligned}
$$

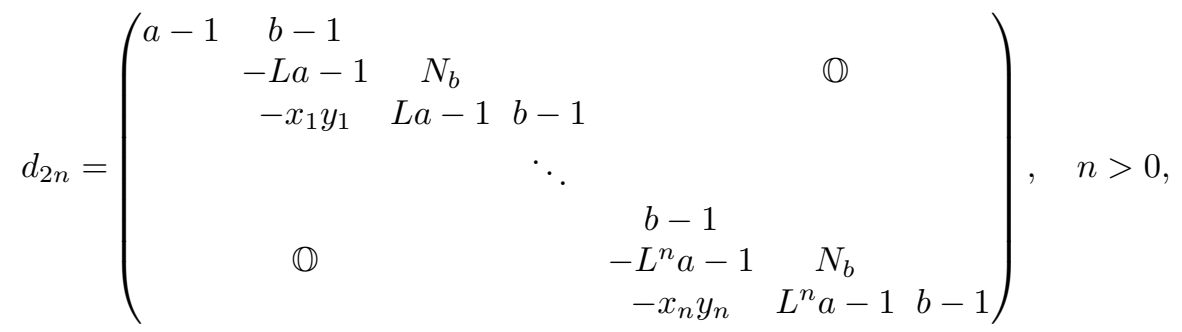

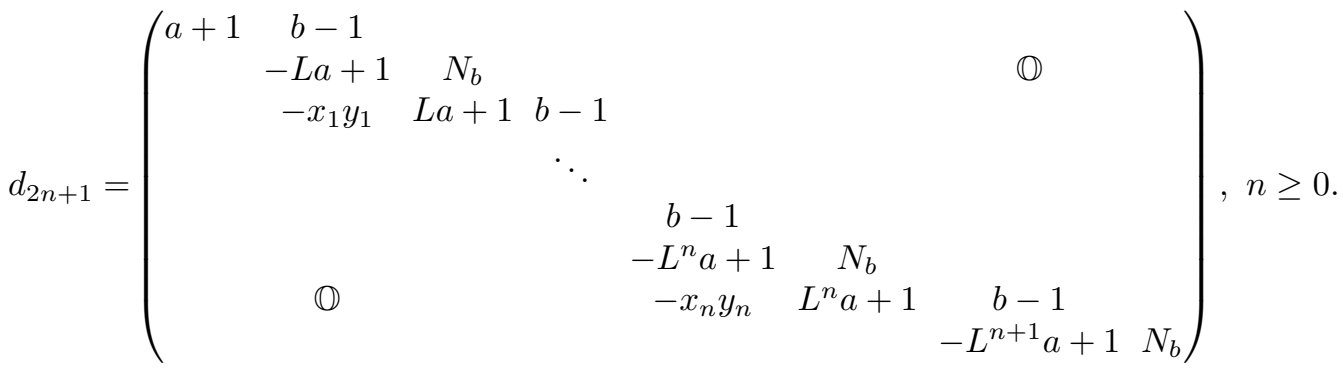

Proposition 1. $Q_{\bullet}=\left(Q_{n}, d_{n}\right)$ is a free resolution of the trivial $R$-module $\mathbb{Z}$.

Proof. This follows from [8, Theorem 1].

We denote by $\Lambda=R^{\mathrm{op}} \otimes R$ the envelope algebra of the algebra $R$. Let $\Delta: R \rightarrow \Lambda$ be an algebra homomorphism defined on the elements of $G$ by the formula $\Delta(g)=g^{-1} \otimes g$. For brevity, we denote the image of $x \in R$ under the map $\Delta$ by $\bar{x} \in \Lambda$. Then we build the complex

$$
0 \longleftarrow R \longleftarrow \nu T_{0} \longleftarrow \bar{d}_{0} T_{1} \longleftarrow \bar{d}_{1} \ldots \longleftarrow T_{i} \stackrel{\bar{d}_{i}}{\longleftarrow} T_{i+1} \longleftarrow \cdots,
$$

where $T_{i}=\Lambda^{i+1}, \nu$ is the multiplication map $(\nu(x \otimes y)=x y$ for any $x, y \in R)$, and the differentials are three-diagonal matrices of the following form:

$$
\begin{aligned}
& \bar{d}_{0}=\left(\begin{array}{ll}
\overline{a-1} \overline{b-1}
\end{array}\right),
\end{aligned}
$$

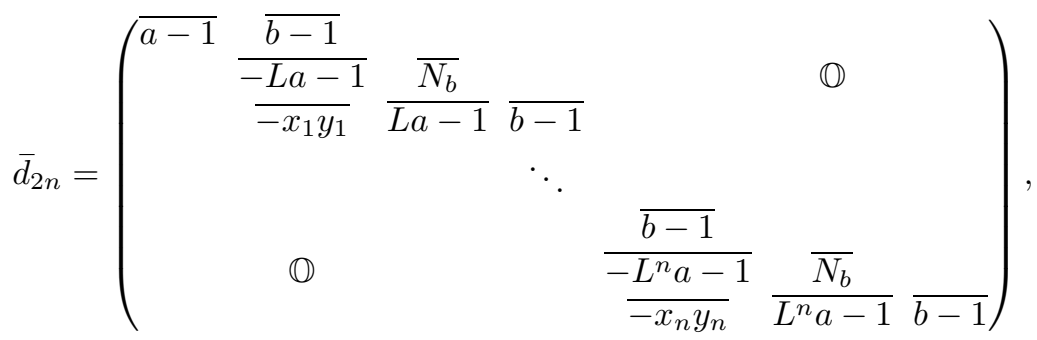




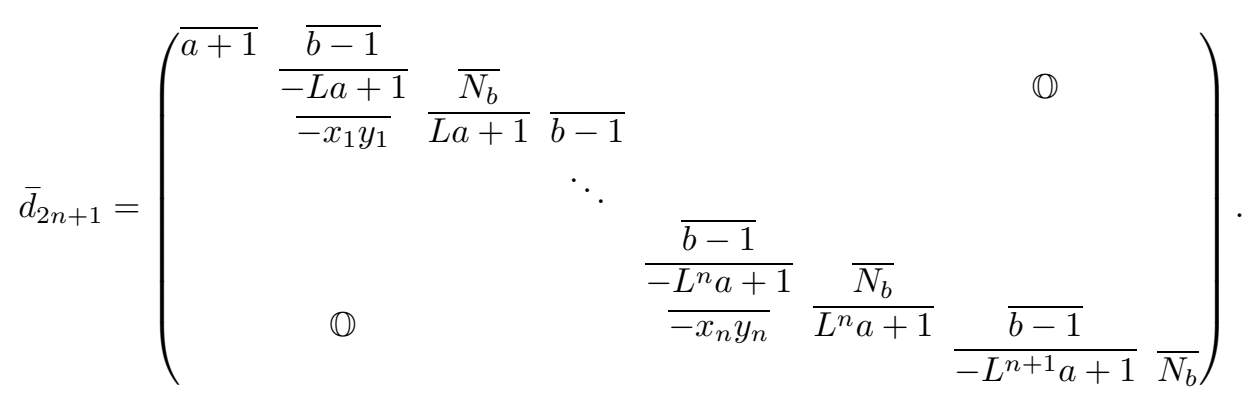

Corollary 1. $T_{\bullet}=\left(T_{n}, \bar{d}_{n}\right)$ is a free resolution of the $\Lambda$-module $R$.

Proof. This follows from Proposition 1 and [9, Theorem 2].

\section{§3. Additive structure}

We denote by $e_{i}^{n}(1 \leq i \leq n+1)$ the element of the module $Q_{n}=R^{n+1}$ that corresponds to the column with 1 at the $i$ th position and with 0 at the other positions. In this section, we compute the groups $\operatorname{HH}^{s}(R)=\operatorname{Ext}_{\Lambda}^{s}(R, R)$.

Theorem 1. For all $s \geq 0$, we have

$$
\mathrm{HH}^{s}(R)= \begin{cases}\mathbb{Z}^{5 r / 2} & \text { if } s=0, \\ \mathbb{Z}_{2}^{2 r k} \oplus \mathbb{Z}_{r}^{r / 2} \oplus \mathbb{Z}_{2 r}^{2 r} & \text { if } s=4 k>0, \\ \mathbb{Z}_{2}^{2 r k} & \text { if } s=4 k+1, \\ \mathbb{Z}_{2}^{(4 k+3) r / 2} \oplus \mathbb{Z}_{r}^{3 r / 2} \oplus \mathbb{Z}_{2 r}^{r} & \text { if } s=4 k+2, \\ \mathbb{Z}_{2}^{(4 k+1) r / 2} & \text { if } s=4 k+3 .\end{cases}
$$

We divide the proof of this theorem into several lemmas. First, we prove a statement having more general nature. Let $G$ be an arbitrary finite group, $K$ a commutative ring with unit, $S=K G$ the corresponding group algebra, and $G^{G}$ a set of representatives of the conjugacy classes of $G$. For $g \in G$, let $C(g)$ be the centralizer of $g$ in $G$, and $O(g)$ the conjugacy class of $g$. We denote by $\widetilde{S}$ an $S$-module that is isomorphic to $S$ as a $K$-module and on which the group $G$ acts by conjugation (i.e., $a * g=g^{-1} a g$ for any $a \in \widetilde{S}$, and $g \in G)$. Let $M^{(g)}$ denote the submodule in $\widetilde{S}$ generated by the set $O(g)$.

Lemma 1. Put $S=K G$ and fix $g \in G$. For any $s \geq 0$, we have

$$
\mathrm{H}^{s}\left(G, M^{(g)}\right) \simeq \mathrm{H}^{s}(C(g), K) .
$$

Proof. Put $H=C(g)$. Assume that $K \stackrel{\varepsilon}{\longrightarrow} Q^{\bullet}$ is a $K H$-injective resolution of the trivial $H$-module $K$. Then

$$
\operatorname{Hom}_{K H}(K G, K) \stackrel{\varepsilon_{*}}{\longrightarrow} \operatorname{Hom}_{K H}\left(K G, Q^{\bullet}\right)
$$

is an injective resolution of the $K G$-module $\operatorname{Hom}_{K H}(K G, K) \simeq M^{(g)}$. Hence,

$$
\begin{aligned}
\mathrm{H}^{s}(H, K) & =\operatorname{Ext}_{K H}^{s}(K, K)=\mathrm{H}^{s}\left(\operatorname{Hom}_{K H}\left(K \otimes_{K G} K G, Q^{\bullet}\right)\right) \\
& \simeq \mathrm{H}^{s}\left(\operatorname{Hom}_{K G}\left(K, \operatorname{Hom}_{K H}\left(K G, Q^{\bullet}\right)\right)\right)=\mathrm{H}^{s}\left(G, M^{(g)}\right) .
\end{aligned}
$$

Corollary 2 ([12, Theorem 2.11.2]). Put $S=K G$. For any $s \geq 0$, we have

$$
\mathrm{HH}^{s}(S) \simeq \bigoplus_{g \in G^{G}} \mathrm{H}^{s}(C(g), K)
$$

Proof. It is well known (see [7, [9, Corollary 3], [13, §5], and [14, Chapter X, Theorem 5.5]) that $\operatorname{HH}^{s}(S) \simeq \mathrm{H}^{s}(G, \widetilde{S})$. It is easily seen that $\widetilde{S} \simeq \bigoplus_{g \in G^{G}} M^{(g)}$, and the claim follows. 
In the sequel, we put $G=M_{2^{\ell}}, R=\mathbb{Z} G$. Let

$$
\tau: \mathrm{HH}^{*}(R) \stackrel{\simeq}{\longrightarrow} \mathrm{H}^{*}(G, \widetilde{R})
$$

denote the isomorphism of Abelian groups considered in Corollary 2 ,

\section{Corollary 3.}

$$
\begin{aligned}
\mathrm{HH}^{s}(R) \simeq\left(\mathrm{H}^{s}(G, \mathbb{Z})\right)^{r} & \oplus\left(\mathrm{H}^{s}\left(G, M^{(a)}\right)\right)^{r / 2} \\
& \oplus\left(\mathrm{H}^{s}\left(G, M^{(b)}\right)\right)^{r / 2} \oplus\left(\mathrm{H}^{s}\left(G, M^{(a b)}\right)\right)^{r / 2} .
\end{aligned}
$$

Proof. This follows immediately from the description of conjugacy classes of the group $G$ :

$$
\begin{array}{rlrl}
O\left(b^{2 i}\right) & =\left\{b^{2 i}\right\} & & \text { for } 0 \leq i \leq r-1, \\
O\left(b^{2 i+1}\right) & =\left\{b^{2 i+1}, b^{2 i+t}\right\} & & \text { for } 0 \leq i \leq \frac{r}{2}-1, \\
O\left(a b^{2 i}\right) & =\left\{a b^{2 i}, a b^{2 i+r}\right\} & \text { for } 0 \leq i \leq \frac{r}{2}-1, \\
O\left(a b^{2 i+1}\right) & =\left\{a b^{2 i+1}, a b^{2 i+t}\right\} & \text { for } 0 \leq i \leq \frac{r}{2}-1,
\end{array}
$$

and, as a consequence, from the isomorphisms

$$
M^{\left(a b^{2 i}\right)} \simeq M^{(a)}, \quad M^{\left(b^{2 i+1}\right)} \simeq M^{(b)}, \quad M^{\left(a b^{2 i+1}\right)} \simeq M^{(a b)} .
$$

Remark 1. In the subsequent calculations it will be convenient to use the decomposition of the form (3.3) instead of (3.1). This is explained by our desire to obtain representatives of cohomology classes in terms of our resolution (2.1).

We introduce some additional notation. For the modules $M^{(a)}, M^{(b)}, M^{(a b)}$, we fix the following $\mathbb{Z}$-bases:

$$
M^{(a)}=\left\langle a+a b^{r}, a\right\rangle, \quad M^{(b)}=\left\langle b+b^{t}, b\right\rangle, \quad M^{(a b)}=\left\langle a\left(b+b^{t}\right), a b\right\rangle .
$$

Then the action of the group $G$ on these bases is defined, respectively, by homomorphisms $\chi_{a}, \chi_{b}, \chi_{a b}: G \rightarrow \mathrm{GL}(2, \mathbb{Z})$, where

$$
\chi_{a}(b)=\chi_{b}(a)=\chi_{a b}(a)=\chi_{a b}(b)=\left(\begin{array}{cc}
1 & 1 \\
0 & -1
\end{array}\right), \quad \chi_{a}(a)=\chi_{b}(b)=\left(\begin{array}{ll}
1 & 0 \\
0 & 1
\end{array}\right) .
$$

We denote by $\chi_{a}, \chi_{b}$, and $\chi_{a b}$ not only the corresponding group homomorphisms, but also the induced maps from $R$ into $\mathrm{M}(2, \mathbb{Z})$.

We denote by $\mathbb{O}_{m}^{n}$ (respectively, by $\mathbb{O}^{n}$ and $\mathbb{O}_{m}$ ) the zero matrix of size $n \times m$ (respectively, the zero column of height $n$, and the zero row of length $m$ ). We consider elements of the free module $\mathbb{Z}^{n}$ as columns of height $n$. Furthermore, if $v$ is a row of length $m$, then we denote by $[v]_{i}^{n}(1 \leq i \leq n-m+1)$ the vector $\left(\mathbb{O}_{i-1}, v, \mathbb{O}_{n-m-i+1}\right)^{\mathrm{T}} \in \mathbb{Z}^{n}$.

If $N$ is an $R$-module, we put

$$
\delta_{N}^{s}=\operatorname{Hom}_{R}\left(d_{s}, N\right): \operatorname{Hom}_{R}\left(Q_{s}, N\right) \rightarrow \operatorname{Hom}_{R}\left(Q_{s+1}, N\right),
$$

where $d_{s}$ is the differential in the resolution (2.1). Moreover, we often identify notationally a cocycle $f \in \operatorname{Ker} \delta_{N}^{s}$ and its cohomology class in $\mathrm{H}^{s}(G, N)$.

Lemma 2 (Cohomology groups). For any $s \geq 0$,

$$
\mathrm{H}^{s}(G, \mathbb{Z}) \simeq \begin{cases}\mathbb{Z}, & \text { if } s=0, \\ \mathbb{Z}_{2}^{k} \oplus \mathbb{Z}_{2 r} & \text { if } s=4 k>0, \\ \mathbb{Z}_{2}^{k} & \text { if } s=4 k+1 \\ \mathbb{Z}_{2}^{k+1} \oplus \mathbb{Z}_{r} & \text { if } s=4 k+2, \\ \mathbb{Z}_{2}^{k} & \text { if } s=4 k+3 .\end{cases}
$$


Proof. It is clear that $\operatorname{Hom}_{R}\left(Q_{i}, \mathbb{Z}\right) \simeq \mathbb{Z}^{i+1}$, and we may assume, modulo this isomorphism, that

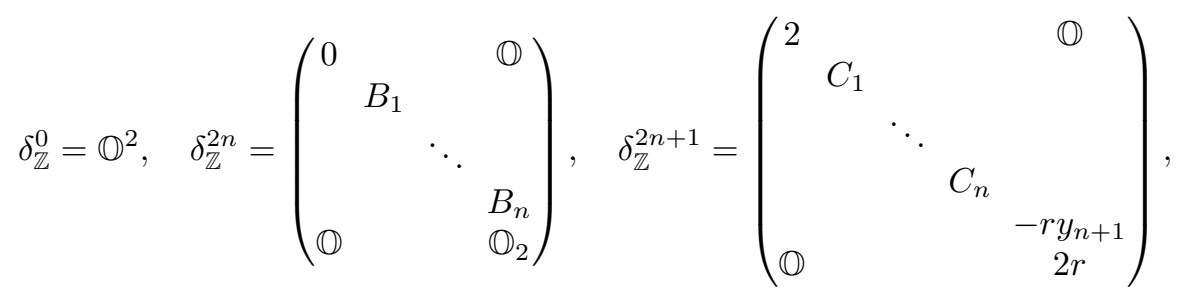

where $B_{k}=\left(\begin{array}{cc}-2 x_{k} & -x_{k} y_{k} \\ 2 r & r y_{k}\end{array}\right)$ and $C_{k}=\left(\begin{array}{cc}-r y_{k} & -x_{k} y_{k} \\ 2 r & 2 x_{k}\end{array}\right)$ (we use the same notation for homomorphisms $\mathbb{Z}^{2} \rightarrow \mathbb{Z}^{2}$ defined by these matrices). It is easily seen that

$$
\begin{aligned}
\operatorname{Ker} B_{2 k-1} & =\left\langle\left(\begin{array}{c}
-y_{2 k-1} \\
2
\end{array}\right)\right\rangle, \quad \operatorname{Ker} B_{2 k}=\left\langle\left(\begin{array}{c}
-x_{k} y_{k} \\
1
\end{array}\right)\right\rangle, \\
\operatorname{Im} B_{2 k-1} & =\left\langle\left(\begin{array}{c}
-x_{2 k-1} \\
r
\end{array}\right)\right\rangle, \quad \operatorname{Im} B_{2 k}=\left\langle\left(\begin{array}{c}
-2 x_{2 k} \\
2 r
\end{array}\right)\right\rangle, \\
\operatorname{Ker} C_{k} & =\left\langle\left(\begin{array}{c}
-x_{k} \\
r
\end{array}\right)\right\rangle, \quad \operatorname{Im} C_{k}=\left\langle\left(\begin{array}{c}
-y_{k} \\
2
\end{array}\right)\right\rangle .
\end{aligned}
$$

We denote

$$
f_{2 n+1, k}=\left[-x_{k}, r\right]_{2 k}^{2 n+2}, \quad f_{2 n, k}= \begin{cases}{[1]_{1}^{2 n+1}} & \text { if } k=0 \\ {\left[-\frac{y_{k}}{2}, 1\right]_{2 k}^{2 n+1}} & \text { if } k: 2, k>0 \\ {\left[-y_{k}, 2\right]_{2 k}^{2 n+1}} & \text { if } k \% 2\end{cases}
$$

Then

$$
\begin{aligned}
\operatorname{Ker} \delta_{\mathbb{Z}}^{2 n} & =\left\langle\left\{f_{2 n, k}\right\}_{0 \leq k \leq n}\right\rangle, \operatorname{Im} \delta_{\mathbb{Z}}^{2 n}=\left\langle\left\{q_{k} f_{2 n+1, k}\right\}_{1 \leq k \leq n}\right\rangle, \\
\operatorname{Ker} \delta_{\mathbb{Z}}^{2 n+1} & =\left\langle\left\{f_{2 n+1, k}\right\}_{1 \leq k \leq n}\right\rangle, \\
\operatorname{Im} \delta_{\mathbb{Z}}^{2 n+1} & =\left\langle\left\{q_{k} f_{2 n+2, k}\right\}_{0 \leq k \leq n}, r q_{n+1} f_{2 n+2, n+1}\right\rangle,
\end{aligned}
$$

where $q_{k}=2$ if $k: 2$, and $q_{k}=1$, if $k \% 2$. Now, the claim is deduced easily.

Lemma 3. For any $s \geq 0$, we have

$$
\mathrm{H}^{s}\left(G, M^{(b)}\right) \simeq \mathrm{H}^{s}\left(G, M^{(a b)}\right) \simeq \begin{cases}\mathbb{Z} & \text { if } s=0 \\ \mathbb{Z}_{2 r} & \text { if } s=2 n>0 \\ 0 & \text { if } s=2 n+1\end{cases}
$$

Furthermore, each of the groups $\mathrm{H}^{0}\left(G, M^{(b)}\right)$ and $\mathrm{H}^{0}\left(G, M^{(a b)}\right)$ is generated by the element of the form $[1]_{1}^{2}$, and for $n>0$, we have

$$
\begin{aligned}
\mathrm{H}^{2 n}\left(G, M^{(b)}\right) & =\left\langle\left[-x_{n} y_{n}, x_{n} y_{n}, 1, r y_{n}\right]_{4 n-1}^{4 n+2}\right\rangle, \\
\mathrm{H}^{2 n}\left(G, M^{(a b)}\right) & =\left\langle\left[-y_{n}, 1\right]_{4 n}^{4 n+2}\right\rangle .
\end{aligned}
$$

Proof. By Lemma 11 formula (3.7) follows from the fact that $C(g) \simeq \mathrm{C}_{2 r}$ for $g \in$ $\{b, a b\}$. The remaining part of the lemma will be discussed in detail only for the groups $\mathrm{H}^{2 n}\left(G, M^{(a b)}\right)$. For simplicity, we denote the module $M^{(a b)}$ by $M$. 
It is easily seen that $\chi_{a b}\left(L^{k} a\right)=\left(\begin{array}{cc}t^{k} & x_{k} \\ 0 & -1\end{array}\right)$ and $\chi_{a b}\left(N_{b}\right)=\left(\begin{array}{cc}2 r & r \\ 0 & 0\end{array}\right)$. Clearly, we have $\operatorname{Hom}_{R}\left(Q_{i}, M\right) \simeq \mathbb{Z}^{2(i+1)}$, and, modulo this isomorphism, we may assume that

$$
\delta_{M}^{0}=\left(\begin{array}{cc}
0 & 1 \\
0 & -2 \\
0 & 1 \\
0 & -2
\end{array}\right), \delta_{M}^{2 n}=\left(\begin{array}{ccccc}
A & & & & \mathbb{0} \\
B^{\prime} & B_{1} & & & \\
& B & B_{2} & & \\
& & \ddots & \ddots & \\
& & & B & B_{n} \\
\mathbb{O} & & & & B^{\prime \prime}
\end{array}\right), \delta_{M}^{2 n+1}=\left(\begin{array}{ccccccc}
A^{\prime} & & & & & & \mathbb{O} \\
B^{\prime} & C_{1} & & & & & \\
& B & C_{2} & & & \\
& & \ddots & \ddots & & \\
& & & B & C_{n} & \\
& & & & B & C_{n+1}^{\prime}
\end{array}\right),
$$

where $A=\left(\begin{array}{cc}0 & 1 \\ 0 & -2\end{array}\right), A^{\prime}=\left(\begin{array}{ll}2 & 1 \\ 0 & 0\end{array}\right), B=\left(\begin{array}{cc}\mathbb{O}_{2}^{2} & A \\ \mathbb{O}_{2}^{2} & \mathbb{O}_{2}^{2}\end{array}\right), B^{\prime}=\left(\begin{array}{c}A \\ \mathbb{O}_{2}^{2}\end{array}\right), B^{\prime \prime}=\left(\begin{array}{ll}\mathbb{O}_{2}^{2} & A\end{array}\right)$,

$$
B_{k}=\left(\begin{array}{cccc}
-2 x_{k} & -x_{k} & -x_{k} y_{k} & 0 \\
0 & 0 & 0 & -x_{k} y_{k} \\
2 r & r & r y_{k} & x_{k} \\
0 & 0 & 0 & -2
\end{array}\right), \quad C_{k}=\left(\begin{array}{cccc}
-r y_{k} & -x_{k} & -x_{k} y_{k} & 0 \\
0 & 2 & 0 & -x_{k} y_{k} \\
2 r & r & 2 x_{k} & x_{k} \\
0 & 0 & 0 & 0
\end{array}\right) \text {, }
$$

and $C_{k}^{\prime}$ is the matrix consisting of the first two columns of the matrix $C_{k}$.

We introduce the following notation:

$$
\begin{aligned}
& f_{2 n, k}^{(a b)}=\left\{\begin{array}{ll}
{[1]_{1}^{4 n+2}} & \text { if } k=0, \\
{\left[-y_{k}, 1\right]_{4 k}^{4 n+2}} & \text { if } k>0,
\end{array} \quad(0 \leq k \leq n),\right. \\
& g_{2 n, k}^{(a b)}=[1,-2]_{4 k-1}^{4 n+2} \quad(1 \leq k \leq n) .
\end{aligned}
$$

It is clear that $\operatorname{Ker} \delta_{M}^{0}=\left\langle f_{0,0}^{(a b)}\right\rangle$. Now we consider the case where $s=2 n>0$. Assume that $v \in \operatorname{Ker} \delta_{M}^{2 n} \subset \operatorname{Hom}_{R}\left(Q_{2 n}, M\right) \simeq \mathbb{Z}^{4 n+2}$. Considering the $(4 m+2)$ nd row of the matrix $\delta_{M}^{2 n}$, for any $0 \leq m \leq n$ we see that $(4 m+2)$ nd element of $v$ is equal to 0 . Then $v=\left[v_{0}\right]_{1}^{4 n+2}+\sum_{k=1}^{n}\left[v_{k}\right]_{4 k-1}^{4 n+2}$, where $v_{0} \in \mathbb{Z}$ and the $v_{k}$ are rows with length 3 $(1 \leq k \leq n)$. Obviously, the condition $v \in \operatorname{Ker} \delta_{M}^{2 n}$ is equivalent to $B_{k}\left(v_{k}, 0\right)^{\mathrm{T}}=0$ for all $1 \leq k \leq n$. If we put $v_{k}=\left(u_{k, 1}, u_{k, 2}, u_{k, 3}\right)$, then, in its turn, the last identity (for $k$ fixed) is equivalent to the relation $2 u_{k, 1}+u_{k, 2}+y_{k} u_{k, 3}=0$. Consequently,

$$
\operatorname{Ker} \delta_{M}^{2 n}=\left\langle\left\{f_{2 n, k}^{(a b)}\right\}_{0 \leq k \leq n},\left\{g_{2 n, k}^{(a b)}\right\}_{1 \leq k \leq n}\right\rangle
$$

and then

(3.8) $\operatorname{Ker} \delta_{M}^{2 n}=\left\langle\left\{f_{2 n, k}^{(a b)}\right\}_{0 \leq k \leq n-2},\left\{g_{2 n, k}^{(a b)}\right\}_{1 \leq k \leq n-1}, f_{2 n, n-1}^{(a b)}+g_{2 n, n}^{(a b)}, g_{2 n, n}^{(a b)}-r f_{2 n, n}^{(a b)}, f_{2 n, n}^{(a b)}\right\rangle$.

It is clear that the set in the angular brackets of the last formula is a set of free generators for $\operatorname{Ker} \delta_{M}^{2 n}$. Hence, it suffices to prove that

$$
\operatorname{Im} \delta_{M}^{2 n-1}=\left\langle\left\{f_{2 n, k}^{(a b)}\right\}_{0 \leq k \leq n-2},\left\{g_{2 n, k}^{(a b)}\right\}_{1 \leq k \leq n-1}, f_{2 n, n-1}^{(a b)}+g_{2 n, n}^{(a b)}, g_{2 n, n}^{(a b)}-r f_{2 n, n}^{(a b)}, 2 r f_{2 n, n}^{(a b)}\right\rangle
$$

First, we note that the columns of the matrix $\delta_{M}^{2 n-1}$ correspond to the elements $2 f_{2 n, 0}^{(a b)}$, $f_{2 n, k}^{(a b)}+g_{2 n, k+1}^{(a b)}(0 \leq k \leq n-1), 2 r f_{2 n, k}^{(a b)}-r y_{k} g_{2 n, k}^{(a b)}, r f_{2 n, k}^{(a b)}-x_{k} g_{2 n, k}^{(a b)}(1 \leq k \leq n)$, and $2 x_{k} f_{2 n, k}^{(a b)}-x_{k} y_{k} g_{2 n, k}^{(a b)}(1 \leq k \leq n-1)$. Since $\operatorname{GCD}\left(x_{k}, r\right)=1$, we see that $2 f_{2 n, k}^{(a b)}-y_{k} g_{2 n, k}^{(a b)} \in$ $\operatorname{Im} \delta_{M}^{2 n-1}$ for $1 \leq k \leq n-1$. Hence, the element

$$
g_{2 n, k}^{(a b)}=\frac{r}{2}\left(2 f_{2 n, k}^{(a b)}-y_{k} g_{2 n, k}^{(a b)}\right)-\left(r f_{2 n, k}^{(a b)}-x_{k} g_{2 n, k}^{(a b)}\right)
$$


lies in the image of $\delta_{M}^{2 n-1}$ for any $1 \leq k \leq n-1$. Consequently, for any $0 \leq k \leq n-2$ we have $f_{2 n, k}^{(a b)} \in \operatorname{Im} \delta_{M}^{2 n-1}$. Next, we observe that

$$
g_{2 n, n}^{(a b)}-r f_{2 n, n}^{(a b)}=\frac{x_{n}+1}{2}\left(2 r f_{2 n, n}^{(a b)}-r y_{n} g_{2 n, n}^{(a b)}\right)-\frac{r y_{n}+2}{2}\left(r f_{2 n, n}^{(a b)}-x_{n} g_{2 n, n}^{(a b)}\right)
$$

and

$$
2 r f_{2 n, n}^{(a b)}=x_{n}\left(2 r f_{2 n, n}^{(a b)}-r y_{n} g_{2 n, n}^{(a b)}\right)-r y_{n}\left(r f_{2 n, n}^{(a b)}-x_{n} g_{2 n, n}^{(a b)}\right) .
$$

Therefore, the set indicated on the right-hand side in (3.9) lies in the image of $\delta_{M}^{2 n-1}$. It remains to prove that this set generates that image. Let $T \subset \operatorname{Im} \delta_{M}^{2 n-1}$ be a submodule generated by this set. Since

$$
2 f_{2 n, n-1}^{(a b)}=2\left(f_{2 n, n-1}^{(a b)}+g_{2 n, n}^{(a b)}\right)-2\left(g_{2 n, n}^{(a b)}-r f_{2 n, n}^{(a b)}\right)-2 r f_{2 n, n}^{(a b)} \in T,
$$

we see that $T$ contains the elements $2 f_{2 n, 0}^{(a b)}, f_{2 n, k}^{(a b)}+g_{2 n, k+1}^{(a b)}(0 \leq k \leq n-1), 2 r f_{2 n, k}^{(a b)}-$ $r y_{k} g_{2 n, k}^{(a b)}, r f_{2 n, k}^{(a b)}-x_{k} g_{2 n, k}^{(a b)}, 2 x_{k} f_{2 n, k}^{(a b)}-x_{k} y_{k} g_{2 n, k}^{(a b)}(1 \leq k \leq n-1)$. It remains to prove that $2 r f_{2 n, n}^{(a b)}-r y_{n} g_{2 n, n}^{(a b)}, r f_{2 n, n}^{(a b)}-x_{n} g_{2 n, n}^{(a b)} \in T$, but this is implied by the following facts: $2 g_{2 n, n}^{(a b)}=2\left(g_{2 n, n}^{(a b)}-r f_{2 n, n}^{(a b)}\right)+2 f_{2 n, n}^{(a b)} \in T$ and $x_{n} \% 2$.

The generators for the groups $\mathrm{H}^{2 n}\left(G, M^{(b)}\right)$ are obtained similarly. In this case, we use the following formulas for $\delta_{M^{(b)}}^{i}$ :

$$
\delta_{M^{(b)}}^{0}=\left(\begin{array}{cc}
0 & 1 \\
0 & -2 \\
0 & 0 \\
0 & 0
\end{array}\right), \quad \delta_{M^{(b)}}^{2 n}=\left(\begin{array}{cccc}
A & & & \mathbb{O} \\
& B_{1} & & \\
& & \ddots & \\
& & & B_{n} \\
\mathbb{O} & & & \mathbb{O}_{4}^{2}
\end{array}\right), \quad \delta_{M^{(b)}}^{2 n+1}=\left(\begin{array}{lllll}
A^{\prime} & & & & \mathbb{O} \\
& C_{1} & & & \\
& & \ddots & & \\
& & C_{n} & \\
& & & C_{n+1}^{\prime}
\end{array}\right),
$$

where $A=\left(\begin{array}{cc}0 & 1 \\ 0 & -2\end{array}\right), A^{\prime}=\left(\begin{array}{ll}2 & 1 \\ 0 & 0\end{array}\right)$,

$$
B_{k}=\left(\begin{array}{cccc}
-2 x_{k} & -t^{k} & -x_{k} y_{k} & 0 \\
0 & r y_{k} & 0 & -x_{k} y_{k} \\
2 r & 0 & r y_{k} & t^{k} \\
0 & 2 r & 0 & -2 x_{k}
\end{array}\right), \quad C_{k}=\left(\begin{array}{cccc}
-r y_{k} & -t^{k} & -x_{k} y_{k} & 0 \\
0 & 2 x_{k} & 0 & -x_{k} y_{k} \\
2 r & 0 & 2 x_{k} & t^{k} \\
0 & 2 r & 0 & -r y_{k}
\end{array}\right),
$$

and $C_{k}^{\prime}$ is the matrix consisting of the first two columns of the matrix $C_{k}$.

Lemma 4. For any $s \geq 0$, we have

$$
\mathrm{H}^{s}\left(G, M^{(a)}\right) \simeq \begin{cases}\mathbb{Z} & \text { if } s=0, \\ \mathbb{Z}_{2}^{n} \oplus \mathbb{Z}_{r} & \text { if } s=2 n>0, \\ \mathbb{Z}_{2}^{n} & \text { if } s=2 n+1 .\end{cases}
$$

Moreover, for the role of generating sets of the groups $\mathrm{H}^{s}\left(G, M^{(a)}\right)$ we can take the following sets:

(a) $\mathrm{H}^{0}\left(G, M^{(a)}\right)$ is generated by the element $[1]_{1}^{2}$;

(b) if $s=4 \ell(\ell>0)$, then the group $\mathrm{H}^{s}\left(G, M^{(a)}\right)$ is generated by the elements

$$
\begin{array}{ll}
{[1]_{1}^{8 \ell+2}, \quad[1,0-1,1]_{2}^{8 \ell+2}, \quad\left[\frac{y_{2 i}}{2}, 0,-1\right]_{8 i-1}^{8 \ell+2}} & \text { for } 1 \leq i \leq \ell, \\
{\left[\frac{-x_{2 i} y_{2 i}}{2}, \frac{r y_{2 i}}{4}, 1, \frac{1-y_{2 i+1}}{2},-1,1\right]_{8 i}^{8 \ell+2}} & \text { for } 1 \leq i \leq \ell-1,
\end{array}
$$

where $\operatorname{ord}\left(\left[\frac{y_{2 \ell}}{2}, 0,-1\right]_{8 \ell-1}^{8 \ell+2}\right)=r$, and the orders of the remaining elements are equal to 2 ; 
(c) if $s=4 \ell+2$, then the group $\mathrm{H}^{s}\left(G, M^{(a)}\right)$ is generated by the elements

$$
\begin{array}{ll}
{[1]_{1}^{8 \ell+6}, \quad[1,0-1,1]_{2}^{8 \ell+6}, \quad\left[\frac{y_{2 i}}{2}, 0,-1\right]_{8 i-1}^{8 \ell+6}} & \text { for } 1 \leq i \leq \ell, \\
{\left[\frac{-x_{2 i} y_{2 i}}{2}, \frac{r y_{2 i}}{4}, 1, \frac{1-y_{2 i+1}}{2},-1,1\right]_{8 i}^{8 \ell+6}} & \text { for } 1 \leq i \leq \ell,
\end{array}
$$

where $\operatorname{ord}\left(\left[\frac{-x_{2 \ell} y_{2 \ell}}{2}, \frac{r y_{2 \ell}}{4}, 1, \frac{1-y_{2 \ell+1}}{2},-1,1\right]_{8 \ell}^{8 \ell+6}\right)=r$, and the orders of the remaining elements are equal to 2 ;

(d) if $s=2 n+1$, then the group $\mathrm{H}^{s}\left(G, M^{(a)}\right)$ is generated by the

$$
\left[-x_{k}, \frac{r}{2}\right]_{4 k}^{4 n+4} \text { for } 1 \leq k \leq n
$$

where the orders of all elements are equal to 2.

Proof. By Lemma 1, formula (3.10) follows from the fact that $C(a) \simeq \mathrm{C}_{r} \times \mathrm{C}_{2}$. Next, it is easily seen that $\chi_{a}\left(L^{k} a\right)=\left(\begin{array}{cc}t^{k} & \frac{r y_{k}}{2} \\ 0 & 1\end{array}\right), \chi_{a}\left(N_{b}\right)=\left(\begin{array}{cc}2 r & r \\ 0 & 0\end{array}\right)$. Clearly, we have $\operatorname{Hom}_{R}\left(Q_{i}, M^{(a)}\right) \simeq \mathbb{Z}^{2(i+1)}$; hence, modulo this isomorphism, we may assume that

$$
\begin{aligned}
& \delta_{M^{(a)}}^{0}=\left(\begin{array}{cc}
0 & 0 \\
0 & 0 \\
0 & 1 \\
0 & -2
\end{array}\right), \quad \delta_{M^{(a)}}^{2 n}=\left(\begin{array}{ccccc}
\mathbb{O}_{2}^{2} & & & & \mathbb{O} \\
B^{\prime} & B_{1} & & & \\
& B & B_{2} & & \\
& & \ddots & \ddots & \\
& & & B & B_{n} \\
\mathbb{O} & & & & B^{\prime \prime}
\end{array}\right), \\
& \delta_{M^{(a)}}^{2 n+1}=\left(\begin{array}{cccccc}
A & & & & & \mathbb{O} \\
B^{\prime} & C_{1} & & & & \\
& B & C_{2} & & & \\
& & \ddots & \ddots & & \\
& & & B & C_{n} & \\
\mathbb{O} & & & & B & C_{n+1}^{\prime}
\end{array}\right),
\end{aligned}
$$

where $A=\left(\begin{array}{ll}2 & 0 \\ 0 & 2\end{array}\right), B=\left(\begin{array}{cc}\mathbb{O}_{2}^{2} & X \\ \mathbb{O}_{2}^{2} & \mathbb{O}_{2}^{2}\end{array}\right), B^{\prime}=\left(\begin{array}{c}X \\ \mathbb{O}_{2}^{2}\end{array}\right), B^{\prime \prime}=\left(\begin{array}{ll}\mathbb{O}_{2}^{2} & X\end{array}\right), X=\left(\begin{array}{cc}0 & 1 \\ 0 & -2\end{array}\right)$,

$$
B_{k}=\left(\begin{array}{cccc}
-2 x_{k} & -\frac{r y_{k}}{2} & -x_{k} y_{k} & 0 \\
0 & -2 & 0 & -x_{k} y_{k} \\
2 r & r & r y_{k} & \frac{r y_{k}}{2} \\
0 & 0 & 0 & 0
\end{array}\right), \quad C_{k}=\left(\begin{array}{cccc}
-r y_{k} & -\frac{r y_{k}}{2} & -x_{k} y_{k} & 0 \\
0 & 0 & 0 & -x_{k} y_{k} \\
2 r & r & 2 x_{k} & \frac{r y_{k}}{2} \\
0 & 0 & 0 & 2
\end{array}\right) \text {, }
$$

and $C_{k}^{\prime}$ is the matrix consisting of the first two columns of the matrix $C_{k}$.

It is clear that $\mathrm{H}^{0}\left(G, M^{(a)}\right)=\left\langle[1]_{1}^{2}\right\rangle$. Now, we study the groups $\mathrm{H}^{2 n}\left(G, M^{(a)}\right), n>0$. For $0 \leq k \leq n$ we put

$$
f_{2 n, k}^{(a)}= \begin{cases}{[1]_{1}^{4 n+2}} & \text { if } k=0 \\ {\left[\frac{y_{k}}{2}, 0,-1\right]_{4 k-1}^{4 n+2}} & \text { if } k: 2, k>0 \\ {\left[y_{k}, 0,-2\right]_{4 k-1}^{4 n+2}} & \text { if } k \% 2\end{cases}
$$

and for $0 \leq k \leq n-1$ we put

$$
g_{2 n, k}^{(a)}= \begin{cases}{[1,0,-1,1]_{2}^{4 n+2}} & \text { if } k=0, \\ {\left[-\frac{x_{k} y_{k}}{2}, \frac{r y_{k}}{4}, 1, \frac{1-y_{k+1}}{2},-1,1\right]_{4 k}^{4 n+2}} & \text { if } k: 2, k>0, \\ {\left[-x_{k} y_{k}, \frac{r y_{k}}{2}, 2,1-y_{k+1},-2,2\right]_{4 k}^{4 n+2}} & \text { if } k \% 2 .\end{cases}
$$


We prove that the set $\left\{f_{2 n, k}^{(a)}\right\}_{0 \leq k \leq n} \cup\left\{g_{2 n, k}^{(a)}\right\}_{0 \leq k \leq n-1}$ freely generates $\operatorname{Ker} \delta_{M^{(a)}}^{2 n}$. It suffices to prove only that the indicated elements generate this module. It is easy to verify that the elements $f_{2 n, k}^{(a)}$ for $0 \leq k \leq n$ and $g_{2 n, k}^{(a)}$ for $0 \leq k \leq n-1$ lie in $\operatorname{Ker} \delta_{M^{(a)}}^{2 n}$. Assume that $v \in \operatorname{Ker} \delta_{M^{(a)}}^{2 n}$ and prove that $v=\sum_{k=0}^{n} \alpha_{k} f_{2 n, k}^{(a)}+\sum_{k=0}^{n-1} \beta_{k} g_{2 n, k}^{(a)}$ for some $\alpha_{k}, \beta_{k} \in \mathbb{Z}$. Obviously, we can choose $\alpha_{0}, \beta_{0}$ such that the first two coordinates of the vector $v-\alpha_{0} f_{2 n, 0}^{(a)}-\beta_{0} g_{2 n, 0}^{(a)}$ are 0 . We use induction on $m$ to prove that for any $0 \leq m \leq n-1$ we can choose numbers $\alpha_{k}, \beta_{k}(0 \leq k \leq m)$ such that the first $4 m+2$ coordinates of the vector $v-\sum_{k=0}^{m} \alpha_{k} f_{2 n, k}^{(a)}-\sum_{k=0}^{m} \beta_{k} g_{2 n, k}^{(a)}$ are 0 . For $m=0$ this statement has already been proved. Let $1 \leq m \leq n-1$, and suppose that the statement in question is proved for $m-1$. There exist $\alpha_{k}, \beta_{k}(0 \leq k \leq m-1)$ such that the first $4 m-2$ coordinates of the vector $w=v-\sum_{k=0}^{m-1} \alpha_{k} f_{2 n, k}^{(a)}-\sum_{k=0}^{m-1} \beta_{k} g_{2 n, k}^{(a)}$ are 0 . Let $w_{i}$ denote the $(4 m-2+i)$ th coordinate of the vector $w(1 \leq i \leq 4)$. Then $2 w_{2}+x_{m} y_{m} w_{4}=0$ and $2 r w_{1}+r w_{2}+r y_{m} w_{3}+\frac{r y_{m}}{2} w_{4}=0$. With the help of these identities, it is easy to show that there exist $\alpha_{m}, \beta_{m} \in \mathbb{Z}$ with

$$
\left(w_{1}, w_{2}, w_{3}, w_{4}\right)= \begin{cases}\alpha_{m}\left(\frac{y_{m}}{2}, 0,-1,0\right)+\beta_{m}\left(0,-\frac{x_{m} y_{m}}{2}, \frac{r y_{m}}{4}, 1\right) & \text { if } m: 2, \\ \alpha_{m}\left(y_{m}, 0,-2,0\right)+\beta_{m}\left(0,-x_{m} y_{m}, \frac{r y_{m}}{2}, 2\right) & \text { if } m \% 2 .\end{cases}
$$

Hence, the first $4 m+2$ coordinates of the vector $v-\sum_{k=0}^{m} \alpha_{k} f_{2 n, k}^{(a)}-\sum_{k=0}^{m} \beta_{k} g_{2 n, k}^{(a)}$ are equal to 0 . So, the induction step is verified. Consequently, we may assume that $v=\left[v_{1}, v_{2}, v_{3}, v_{4}\right]_{4 n-1}^{4 n+2}$ for some $v_{1}, v_{2}, v_{3}, v_{4} \in \mathbb{Z}$. Now, considering the rows of the matrix $\delta_{M^{(a)}}^{2 n}$ with numbers $4 n-2,4 n-1$, and $4 n+1$, we easily check that $v_{2}=v_{4}=0$, $2 v_{1}+y_{n} v_{3}=0$, whence, $v=\alpha_{n} f_{2 n, n}^{(a)}$ with $\alpha_{n} \in \mathbb{Z}$. Then we observe that the columns of the matrix $\delta_{M^{(a)}}^{2 n-1}$ correspond to the elements $2 f_{2 n, 0}^{(a)}, q_{k} g_{2 n, k}^{(a)}+q_{k+1} f_{2 n, k+1}^{(a)}(0 \leq k \leq n-1)$, $r q_{k} f_{2 n, k}^{(a)}, \frac{r}{2} q_{k} f_{2 n, k}^{(a)}(1 \leq k \leq n)$, and $x_{k} q_{k} f_{2 n, k}^{(a)}(1 \leq k \leq n-1)$, where $q_{k}=2$ if $k: 2$, and $q_{k}=1$ if $k \% 2$. Since $x_{k} \% 2$, it follows that

$$
\begin{aligned}
\operatorname{Im} \delta_{M^{(a)}}^{2 n-1}= & \left\langle\left\{q_{k} f_{2 n, k}^{(a)}\right\}_{0 \leq k \leq n-1},\left\{q_{k} g_{2 n, k}^{(a)}+q_{k+1} f_{2 n, k+1}^{(a)}\right\}_{0 \leq k \leq n-1}, \frac{r}{2} q_{n} f_{2 n, n}^{(a)}\right\rangle \\
= & \left\langle\left\{q_{k} f_{2 n, k}^{(a)}\right\}_{0 \leq k \leq n-1},\left\{q_{k} g_{2 n, k}^{(a)}\right\}_{0 \leq k \leq n-2}, q_{n-1} g_{2 n, n-1}^{(a)}+q_{n} f_{2 n, n}^{(a)}, \frac{r}{2} q_{n} f_{2 n, n}^{(a)}\right\rangle \\
= & \begin{cases}\left\langle\left\{q_{k} f_{2 n, k}^{(a)}\right\}_{0 \leq k \leq n-1},\left\{q_{k} g_{2 n, k}^{(a)}\right\}_{0 \leq k \leq n-2},\right. & \text { if } n \vdots 2, \\
\left.g_{2 n, n-1}^{(a)}+2 f_{2 n, n}^{(a)}, r f_{2 n, n}^{(a)}\right\rangle & \text { if } n \% 2 . \\
\left\langle q_{k} f_{2 n, k}^{(a)}\right\}_{0 \leq k \leq n-1},\left\{q_{k} g_{2 n, k}^{(a)}\right\}_{0 \leq k \leq n-2}, & \\
\left.2 g_{2 n, n-1}^{(a)}+f_{2 n, n}^{(a)}, r g_{2 n, n-1}^{(a)}\right\rangle & \end{cases}
\end{aligned}
$$

Observe that the sets

$$
\left\{f_{2 n, k}^{(a)}\right\}_{0 \leq k \leq n-1} \cup\left\{g_{2 n, k}^{(a)}\right\}_{0 \leq k \leq n-2} \cup\left\{g_{2 n, n-1}^{(a)}+2 f_{2 n, n}^{(a)}, f_{2 n, n}^{(a)}\right\},
$$

and

$$
\left\{f_{2 n, k}^{(a)}\right\}_{0 \leq k \leq n-1} \cup\left\{g_{2 n, k}^{(a)}\right\}_{0 \leq k \leq n-2} \cup\left\{2 g_{2 n, n-1}^{(a)}+f_{2 n, n}^{(a)}, g_{2 n, n-1}^{(a)}\right\}
$$

are free generating sets for $\operatorname{Ker} \delta_{M^{(a)}}^{2 n}$. Hence, we arrive at the generating sets for the groups $\mathrm{H}^{2 n}\left(G, M^{(a)}\right)$ indicated in items (b) and (c).

Now we study the groups $\mathrm{H}^{2 n+1}\left(G, M^{(a)}\right)$. We put

$$
\begin{array}{ll}
f_{2 n+1, k}^{(a)}=\left[-x_{k}, \frac{r}{2}\right]_{4 k}^{4 n+4} & \text { for } 1 \leq k \leq n, \\
g_{2 n+1, k}^{(a)}=[1,-2]_{4 k-1}^{4 n+4} & \text { for } 1 \leq k \leq n+1 .
\end{array}
$$


Assume that $v \in \operatorname{Ker} \delta_{M^{(a)}}^{2 n+1} \subset \operatorname{Hom}_{R}\left(Q_{2 n+1}, M^{(a)}\right) \simeq \mathbb{Z}^{4 n+4}$. Considering the rows of the matrix $\delta_{M^{(a)}}^{2 n+1}$ with numbers 1 and $4 m+2$ for all $0 \leq m \leq n$, we see that the components of the vector $v$ with there numbers are 0 . Hence, $v=\sum_{k=1}^{n}\left[v_{k}\right]_{4 k-1}^{4 n+2}+\left[v_{0}\right]_{4 n+3}^{4 n+4}$, where the $v_{k}$ are rows of length $3(1 \leq k \leq n)$, and $v_{0}$ is a row of length 2 . It is clear that the condition $v \in \operatorname{Ker} \delta_{M^{(a)}}^{2 n+1}$ is equivalent to $C_{n+1} v_{0}^{\mathrm{T}}=0, C_{k}\left(v_{k}, 0\right)^{\mathrm{T}}=0$ for all $1 \leq k \leq n$. Then it is easy to check (cf. the proof of Lemma 3) that

$$
\operatorname{Ker} \delta_{M^{(a)}}^{2 n+1}=\left\langle\left\{g_{2 n+1, k}^{(a)}\right\}_{1 \leq k \leq n+1} \cup\left\{f_{2 n+1, k}^{(a)}\right\}_{1 \leq k \leq n}\right\rangle .
$$

Clearly, the columns of the matrix $\delta_{M^{(a)}}^{2 n}$ correspond to the elements

$$
\left.\begin{array}{l}
g_{2 n+1,1}^{(a)}, 4 f_{2 n+1, k}^{(a)}-2 x_{k} g_{2 n+1, k}^{(a)}, 2 f_{2 n+1, k}^{(a)}-\frac{r y_{k}}{2} g_{2 n+1, k}^{(a)}, \\
2 y_{k} f_{2 n+1, k}^{(a)}-x_{k} y_{k} g_{2 n+1, k}^{(a)}, y_{k} f_{2 n+1, k}^{(a)}+g_{2 n+1, k+1}^{(a)}, \quad \text { where } 1 \leq k \leq n .
\end{array}\right\}
$$

Put

$$
\left.X=\left\{g_{2 n+1, k}^{(a)}+\kappa_{k-1} f_{2 n+1, k-1}^{(a)}\right)\right\}_{1 \leq k \leq n+1} \cup\left\{2 f_{2 n+1, k}^{(a)}\right\}_{1 \leq k \leq n},
$$

where we assume that $f_{2 n+1,0}^{(a)}=0$, and $\kappa_{k}$ is the remainder of division of $k$ by 2 . Since $\left\{g_{2 n+1, k}^{(a)}+\kappa_{k-1} f_{2 n+1, k-1}^{(a)}\right\}_{1 \leq k \leq n+1} \cup\left\{f_{2 n+1, k}^{(a)}\right\}_{1 \leq k \leq n}$ is a free generating set for $\operatorname{Ker} \delta_{M^{(a)}}^{2 n+1}$, it suffices to verify that $X$ generates $\operatorname{Im} \delta_{M^{(a)}}^{2 n}$. Put

$$
\begin{gathered}
\left.X_{m}=\left\{g_{2 n+1, k}^{(a)}+\kappa_{k-1} f_{2 n+1, k-1}^{(a)}\right)\right\}_{1 \leq k \leq m+1} \cup\left\{2 f_{2 n+1, k}^{(a)}\right\}_{1 \leq k \leq m}, \\
X_{m}^{\prime}=\bigcup_{k=m+1}^{n}\left\{4 f_{2 n+1, k}^{(a)}-2 x_{k} g_{2 n+1, k}^{(a)}, 2 f_{2 n+1, k}^{(a)}-\frac{r y_{k}}{2} g_{2 n+1, k}^{(a)}\right. \\
\left.2 y_{k} f_{2 n+1, k}^{(a)}-x_{k} y_{k} g_{2 n+1, k}^{(a)}, y_{k} f_{2 n+1, k}^{(a)}+g_{2 n+1, k+1}^{(a)}\right\} .
\end{gathered}
$$

Let $T_{m} \subset \mathbb{Z}^{4 n+4}$ denote the submodule generated by $X_{m} \cup X_{m}^{\prime}$. We shall show that $T_{m}=\operatorname{Im} \delta_{M^{(a)}}^{2 n}$ for any $0 \leq m \leq n$. Since $X_{n}=X$ and $X_{n}^{\prime}=\varnothing$, the desired claim will then follow. We have proved that $T_{0}=\operatorname{Im} \delta_{M^{(a)}}^{2 n}$ (see (3.11)). We check that $T_{m}=T_{m-1}$ $(1 \leq m \leq n)$. We need to show that $2 f_{2 n+1, m}^{(a)}, g_{2 n+1, m+1}^{(a)}+\kappa_{m} f_{2 n+1, m}^{(a)} \in T_{m-1}$, and

$$
\begin{aligned}
& 4 f_{2 n+1, m}^{(a)}-2 x_{m} g_{2 n+1, m}^{(a)}, 2 f_{2 n+1, m}^{(a)}-\frac{r y_{m}}{2} g_{2 n+1, m}^{(a)}, \\
& 2 y_{m} f_{2 n+1, m}^{(a)}-x_{m} y_{m} g_{2 n+1, m}^{(a)}, y_{m} f_{2 n+1, m}^{(a)}+g_{2 n+1, m+1}^{(a)} \in T_{m},
\end{aligned}
$$

which is verified easily by using the fact that $2 g_{2 n+1, m}^{(a)} \in T_{m-1}$ and the fact that the numbers $m, y_{m}$ have the same parity.

Consequently, Corollary 3 to Lemma 1 and Lemmas 2,4 immediately imply Theorem 1 .

$$
\S 4 . \mathrm{HH}^{*}(R) \text { AND } \mathrm{H}^{*}(G, \widetilde{R})
$$

As above, $Q . \stackrel{\varepsilon}{\longrightarrow} \mathbb{Z}$ denotes the free $R$-resolution described in $\S 2$. For $R$-modules $N_{1}, N_{2}$, we view $N_{1} \otimes N_{2}$ as an $R$-module with the action of $g \in G$ determined by the rule $\left(n_{1} \otimes n_{2}\right) g=\left(n_{1} g\right) \otimes\left(n_{2} g\right)$. As was mentioned above (see (3.2)), there is an isomorphism $\tau$ : $\mathrm{HH}^{*}(R) \simeq \mathrm{H}^{*}(G, \widetilde{R})$. We can define the $\cup$-product on $\mathrm{HH}^{*}(R)$ and $\mathrm{H}^{*}(G, \widetilde{R})$. $\mathrm{HH}^{*}(R)$ is an algebra with respect to this product. The remaining part of the paper is devoted to the description of this algebra. For this, we need the following description of the $\cup$-product. Assume that $f \in \mathrm{H}^{n}(G, \widetilde{R})$ and $g \in \mathrm{H}^{m}(G, \widetilde{R})$. There exist homomorphisms of $R$-modules $\phi_{i}: Q_{m+i} \rightarrow Q_{i} \otimes \widetilde{R}(i \geq 0)$ such that $\left(\varepsilon \otimes \operatorname{id}_{\widetilde{R}}\right) \phi_{0} \in \operatorname{Ker} \delta_{\widetilde{R}}^{n},\left(\varepsilon \otimes \operatorname{id}_{\widetilde{R}}\right) \phi_{0}=g$ in $\mathrm{H}^{m}(G, \widetilde{R}),\left(d_{i} \otimes \mathrm{id}_{\widetilde{R}}\right) \phi_{i+1}=\phi_{i} d_{i+m}$ (here the 
notation is as in (2.1)). We call $\phi_{i}$ an $i$ th translate of $g$ and denote it by $T^{i}(g)$. Since $\widetilde{R}$ coincides with $R$ as a $\mathbb{Z}$-module, we can introduce a multiplication $\mu: \widetilde{R} \otimes \widetilde{R} \rightarrow \widetilde{R}$ on $\widetilde{R}$ that coincides with the multiplication on $R$. It is easily seen that $\mu$ is a homomorphism of $R$-modules. Then the product of elements $\tau^{-1}(f), \tau^{-1}(g)$ can be calculated by the formula

$$
\tau^{-1}(f) \cup \tau^{-1}(g)=\tau^{-1}(\mu \circ(f \cup g))=\tau^{-1}\left(\mu \circ\left(f \otimes \mathrm{id}_{\widetilde{R}}\right) \circ T^{n}(g)\right) .
$$

The first identity follows from [7, Proposition 3.1] and the second from [15, Chapter 5, Theorem 4.6]. We shall view $\mathrm{H}^{*}(G, \widetilde{R})$ as an algebra with respect to the multiplication obtained from the $\cup$-product on $\mathrm{HH}^{*}(R)$ via the isomorphism $\tau$.

Denote by $\mathbb{Z}(i)(0 \leq i \leq r-1)$ the $R$-submodule of $\widetilde{R}$ generated by $b^{2 i}$, and by $M^{(a)}(i), M^{(b)}(i)$, and $M^{(a b)}(i)\left(0 \leq i \leq \frac{r}{2}-1\right)$ the submodules generated by $a b^{2 i}, b^{2 i+1}$, and $a b^{2 i+1}$, respectively. Clearly, $\mathbb{Z}(i) \simeq \mathbb{Z}, M^{(a)}(i) \simeq M^{(a)}, M^{(b)}(i) \simeq M^{(b)}, M^{(a b)}(i) \simeq$ $M^{(a b)}$. Furthermore, we fix the following $\mathbb{Z}$-bases for $M^{(a)}(i), M^{(b)}(i), M^{(a b)}(i)$ :

$$
\begin{gathered}
M^{(a)}(i)=\left\langle a\left(b^{2 i}+b^{2 i+r}\right), a b^{2 i}\right\rangle, M^{(b)}(i)=\left\langle b^{2 i+1}+b^{2 i+t}, b^{2 i+1}\right\rangle, \\
M^{(a b)}(i)=\left\langle a\left(b^{2 i+1}+b^{2 i+t}\right), a b^{2 i+1}\right\rangle .
\end{gathered}
$$

In this notation, the decomposition (3.3) is written in the form

$$
\tilde{R}=\bigoplus_{i=0}^{r-1} \mathbb{Z}(i) \oplus \bigoplus_{i=0}^{\frac{r-2}{2}} M^{(a)}(i) \oplus \bigoplus_{i=0}^{\frac{r-2}{2}} M^{(b)}(i) \oplus \bigoplus_{i=0}^{\frac{r-2}{2}} M^{(a b)}(i) .
$$

If $N$ is a summand in this decomposition, we write the elements of $\operatorname{Hom}_{R}(R, N) \simeq N$ with respect to the $\mathbb{Z}$-bases indicated above.

Moreover, if $f \in \operatorname{Ker} \delta_{\widetilde{R}}^{n}$ and $\operatorname{Im} f \subset N$, then we can construct $T^{i}(f)(i \geq 0)$ so that $\operatorname{Im} T^{i}(f) \subset Q_{i} \otimes N$. In these cases, we shall construct $T^{i}(f)$ as maps from $Q_{n+i}$ into $Q_{i} \otimes N$.

We pick the following elements of $\mathrm{H}^{*}(G, \widetilde{R})$ :

- $v_{1}, v_{2} \in \mathrm{H}^{2}(G, \mathbb{Z}(0)), v_{1}=[1]_{1}^{3}, v_{2}=[-1,2]_{2}^{3}$,

- $\nu \in \mathrm{H}^{4}(G, \mathbb{Z}(0)), \nu=\left[-x_{1}, 1\right]_{4}^{5}$,

- $\omega \in \mathrm{H}^{5}(G, \mathbb{Z}(0)), \omega=\left[-x_{2}, r\right]_{4}^{6}$,

- $\lambda \in \mathrm{H}^{0}(G, \mathbb{Z}(1)), \lambda=(1)$,

- $\theta_{0} \in \mathrm{H}^{0}\left(G, M^{(a)}(0)\right), \theta_{0}=[1]_{1}^{2}$,

- $\theta_{1} \in \mathrm{H}^{2}\left(G, M^{(a)}(0)\right), \theta_{1}=[1,0,-1,1]_{2}^{6}$,

- $\varphi_{0} \in \mathrm{H}^{0}\left(G, M^{(b)}(0)\right), \varphi_{0}=[1]_{1}^{2}$,

- $\varphi_{1} \in \mathrm{H}^{2}\left(G, M^{(b)}(0)\right), \varphi_{1}=\left[-x_{1}, x_{1}, 1, r\right]_{3}^{6}$,

- $\rho_{1} \in \mathrm{H}^{3}\left(G, M^{(a)}(0)\right), \rho_{1}=\left[-x_{1}, \frac{r}{2}\right]_{4}^{8}$,

- $\rho_{2} \in \mathrm{H}^{5}\left(G, M^{(a)}(0)\right), \rho_{2}=\left[-x_{2}, \frac{r}{2}\right]_{8}^{12}$,

- $\psi_{0} \in \mathrm{H}^{0}\left(G, M^{(a b)}(0)\right), \psi_{0}=[1]_{1}^{2}$,

- $\psi_{1} \in \mathrm{H}^{2}\left(G, M^{(a b)}(0)\right), \psi_{1}=[-1,1]_{4}^{6}$.

The proofs of Lemmas 24 show that all these elements are indeed represented by cocycles of the corresponding degrees.

\section{$\S 5$. Generators}

In this section, we prove that the images under $\tau^{-1}$ (see (3.2)) of the elements presented in the final part of the previous section generate the algebra $\mathrm{HH}^{*}(R)$. To prove this, we need the following lemma. 
Lemma 5. 1) For the role of the translates of the element $v_{1}$, we can take the maps given by the matrices

$$
T^{k}\left(v_{1}\right)=\left(\begin{array}{ll}
I_{k+1} & \mathbb{O}_{2}^{k+1}
\end{array}\right),
$$

where $I_{k+1}$ is the unit matrix of size $(k+1) \times(k+1)$.

2) The translates of $\nu$ for $k \geq 1$ can be chosen so that they are given by the matrices of the form

$$
T^{k}(\nu)=\left(\begin{array}{ccc} 
& \mathbb{O}^{k-1} & \mathbb{O}^{k-1} \\
A_{k}^{\prime} & a L^{2} a & 0 \\
& s_{k} & 1
\end{array}\right),
$$

where $A_{k}^{\prime}$ is a matrix of size $(k+1) \times(k+3), s_{k}=-x_{1}$ if $k$ is even, and $s_{k}=s$ if $k$ is odd; here $s \in R$ is an element satisfying $(b-1) s=s(b-1)=a L^{2} a-1-x_{1} N_{b}$.

Proof. 1) It suffices to prove that $\varepsilon \circ T^{0}\left(v_{1}\right)=v_{1}$ and $d_{i} \circ T^{i+1}\left(v_{1}\right)=T^{i}\left(v_{1}\right) \circ d_{i+2}$ $(i \geq 0)$. This verification is not difficult, and we leave it to the reader.

2) First, we claim that there exists $s \in R$ for which we have $a L^{2} a-1-x_{1} N_{b}=$ $(b-1) s=s(b-1)$. Indeed, this is derived from the identity $\left(a L^{2} a-1-x_{1} N_{b}\right) N_{b}=0$ and the structure of the 2-periodic resolution for the group $\langle b\rangle$.

Clearly, we can take $T^{0}(\nu)=\left(\mathbb{O}_{3}-x_{1} 1\right)$. We must show that there exists a matrix with the last two columns equal to $\left(\begin{array}{lll}a L^{2} a & s\end{array}\right)^{\mathrm{T}}$ and $\left(\begin{array}{ll}0 & 1\end{array}\right)^{\mathrm{T}}$ and such that it determines a map $T^{1}(\nu)$ satisfying $d_{0} T^{1}(\nu)=T^{0}(\nu) d_{4}$. The last condition is equivalent to the following two equations: $(a-1 \quad b-1)\left(\begin{array}{l}0 \\ 1\end{array}\right)=b-1$ (and this is clear), and

$$
\left(\begin{array}{ll}
a-1 & b-1
\end{array}\right)\left(\begin{array}{c}
a L^{2} a \\
s
\end{array}\right)=\left(\begin{array}{ll}
-x_{1} & 1
\end{array}\right)\left(\begin{array}{c}
N_{b} \\
L^{2} a-1
\end{array}\right),
$$

which is verified easily.

Next, we prove the statement of the lemma by induction on $k$. Assume that $k>1$ and

$$
T^{k-1}(\nu)=\left(\begin{array}{ccc} 
& \mathbb{O}^{k-2} & \mathbb{O}^{k-2} \\
A_{k-1}^{\prime} & a L^{2} a & 0 \\
& s_{k-1} & 1
\end{array}\right) .
$$

We check that we can find a matrix $T^{k}(\nu)$ of the desired form, i.e., a matrix with the last two columns equal to $\left(\mathbb{O}_{k-1} \quad a L^{2} a s_{k}\right)^{\mathrm{T}},\left(\begin{array}{ll}\mathbb{O}_{k} & 1\end{array}\right)^{\mathrm{T}}$, such that it determines a map satisfying $d_{k-1} T^{k}(\nu)=T^{k-1}(\nu) d_{k+3}$. If $k=2 n$ with $n>0$, then this claim is equivalent to the identity

$$
\left(\begin{array}{cc}
b-1 & 0 \\
1-L^{n} a & N_{b}
\end{array}\right)\left(\begin{array}{cc}
a L^{2} a & 0 \\
-x_{1} & 1
\end{array}\right)=\left(\begin{array}{cc}
a L^{2} a & 0 \\
s & 1
\end{array}\right)\left(\begin{array}{cc}
b-1 & 0 \\
1-L^{n+2} a & N_{b}
\end{array}\right)
$$

If $k=2 n+1$ with $n>0$, then the same claim is equivalent to the identity

$$
\left(\begin{array}{cc}
N_{b} & 0 \\
L^{n} a-1 & b-1
\end{array}\right)\left(\begin{array}{cc}
a L^{2} a & 0 \\
s & 1
\end{array}\right)=\left(\begin{array}{cc}
a L^{2} a & 0 \\
-x_{1} & 1
\end{array}\right)\left(\begin{array}{cc}
N_{b} & 0 \\
L^{n+2} a-1 & b-1
\end{array}\right) .
$$

The verification of these two relations is easy, and we leave it to the reader.

Proposition 2. The set

$$
\mathcal{X}=\left\{v_{1}, v_{2}, \nu, \omega, \lambda, \varphi_{0}, \varphi_{1}, \theta_{0}, \theta_{1}, \rho_{1}, \rho_{2}, \psi_{0}, \psi_{1}\right\}
$$

generates the algebra $\mathrm{H}^{*}(G, \widetilde{R})$. In other words, the image of this set under $\tau^{-1}$ generates $\mathrm{HH}^{*}(R)$ as an algebra with respect to the $\cup$-product. 
Proof. Let $\mathcal{T}$ denote the subalgebra of $\mathrm{H}^{*}(G, \widetilde{R})$ generated by the set $\mathcal{X}$. We shall prove that $\mathrm{H}^{*}(G, \widetilde{R}) \subset \mathcal{T}$. First, we check that multiplication by $\lambda$ induces isomorphisms $\mathrm{H}^{*}(G, \mathbb{Z}(i)) \rightarrow \mathrm{H}^{*}(G, \mathbb{Z}(i+1))(0 \leq i \leq r-2), \mathrm{H}^{*}\left(G, M^{(a)}(i)\right) \rightarrow \mathrm{H}^{*}\left(G, M^{(a)}(i+1)\right)$, $\mathrm{H}^{*}\left(G, M^{(b)}(i)\right) \rightarrow \mathrm{H}^{*}\left(G, M^{(b)}(i+1)\right)$, and $\mathrm{H}^{*}\left(G, M^{(a b)}(i)\right) \rightarrow \mathrm{H}^{*}\left(G, M^{(a b)}(i+1)\right)(0 \leq$ $\left.i \leq \frac{r-4}{2}\right)$. Indeed, assume that $f: Q_{n} \rightarrow \widetilde{R}$ takes an element $e_{i}^{n}(1 \leq i \leq n+1)$ to $\sum_{g \in G} a_{i, g} g$ with $a_{i, g} \in \mathbb{Z}$. Then for the role of a representative of $\lambda f$ we can choose a map from $Q_{n}$ to $\widetilde{R}$ that takes $e_{i}^{n}$ to $\sum_{g \in G} a_{i, g} g b^{2}$. The isomorphisms in question are obtained with the help of considering $\mathbb{Z}$-bases for the modules $\mathbb{Z}(i), M^{(a)}(i), M^{(b)}(i)$, and $M^{(a b)}(i)$. It remains to show that $\mathrm{H}^{*}(G, \mathbb{Z}(0)), \mathrm{H}^{*}\left(G, M^{(a)}(0)\right), \mathrm{H}^{*}\left(G, M^{(b)}(0)\right)$, and $\mathrm{H}^{*}\left(G, M^{(a b)}(0)\right)$ lie in $\mathcal{T}$. Let $N$ denote an $R$-module with a basis $B$ consisting of $m$ elements. The first part of Lemma 5 implies that if $h$ is a row of length $m(n+1)$ such that $[h]_{1}^{m(n+1)} \in \mathrm{H}^{n}(G, N)$, then

$$
v_{1}[h]_{1}^{m(n+1)}=[h]_{1}^{m(n+3)} .
$$

Let $\mathcal{U}$ be a subalgebra of $\mathrm{H}^{*}(G, \mathbb{Z}(0))$ generated by the elements $v_{1}, v_{2}, \nu$, and $\omega$. We claim that $\mathrm{H}^{*}(G, \mathbb{Z}(0)) \subset \mathcal{U}$. Clearly, the product of elements of $\mathrm{H}^{*}(G, \mathbb{Z}(0))$ obtained by transferring the $\cup$-product on $\mathrm{HH}^{*}(R)$ by using $\tau^{-1}$ coincides with the usual $\cup$-product on $\mathrm{H}^{*}(G, \mathbb{Z}(0)) \simeq \mathrm{H}^{*}(G, \mathbb{Z})$. First, we prove that $\mathrm{H}^{2 n}(G, \mathbb{Z}(0)) \subset \mathcal{U}$ for $n \geq 0$. If $n \in\{0,1\}$, this follows from (3.6) and the definition of $v_{1}, v_{2}$. Then we use induction on $n$. Assume that $\mathrm{H}^{2(n-1)}(G, \mathbb{Z}(0)) \subset \mathcal{U}$. From (5.2) we see that the elements $v_{1} f_{2 n-2,2 k}=f_{2 n, k}$ of $\mathrm{H}^{2 n}(G, \mathbb{Z}(0)), 0 \leq k \leq \frac{n-1}{2}$, lie in $\mathcal{U}$ (see the notation in (3.5)). Using part 2) of Lemma 5 , we easily derive by induction on $k$ that $\nu^{k}$ can be presented in $\mathbb{Z}^{4 k+1}$ by a vector with the last coordinate equal to 1 , and that $v_{2} \nu^{k}$ can be presented in $\mathbb{Z}^{4 k+3}$ by a vector with the last coordinate equal to 2. From this and (3.6), it follows that if $n: 2$, then $\nu^{\frac{n}{2}}=$ $f_{2 n, n}+\sum_{k=0}^{\frac{n-2}{2}} a_{k} f_{2 n, 2 k}$ in $\mathrm{H}^{2 n}(G, \mathbb{Z}(0))$, and if $n \% 2$, then $v_{2} \nu^{\frac{n-1}{2}}=f_{2 n, n}+\sum_{k=0}^{\frac{n-1}{2}} a_{k} f_{2 n, 2 k}$, where all $a_{k}$ are integers. This shows that $\mathrm{H}^{2 n}(G, \mathbb{Z}(0)) \subset \mathcal{U}$. Next, we check that $\mathrm{H}^{2 n+1}(G, \mathbb{Z}(0)) \subset \mathcal{U}$ for $n \geq 0$. If $n=0,1,2$, this follows from (3.6) and the definition of $\omega$. Then we argue by induction on $n$. Assume that $\mathrm{H}^{2 n-1}(G, \mathbb{Z}(0)) \subset \mathcal{U}$. From (5.2) we see that the elements $v_{1} f_{2 n-1,2 k}=f_{2 n+1,2 k}$ of $\mathrm{H}^{2 n+1}(G, \mathbb{Z}(0))\left(1 \leq k \leq \frac{n-1}{2}\right)$ lie in $\mathcal{U}$. If $n \% 2$, then (3.6) immediately implies that $\mathrm{H}^{2 n+1}(G, \mathbb{Z}(0)) \subset \mathcal{U}$. Suppose $n \vdots 2$. Using part 2) of Lemma 5 and the fact that now we have $2 f_{2 n+1, n} \in \operatorname{Im} \delta_{\mathbb{Z}}^{2 n}$, we prove by induction on $k$ that $\omega \nu^{k}$ can be presented by a vector the last but one coordinate of which is equal to $r$, and the last coordinate is zero. Consequently, in $\mathrm{H}^{2 n+1}(G, \mathbb{Z}(0))$ we obtain the identity $\omega \nu^{\frac{n-2}{2}}=f_{2 n+1, n}+\sum_{k=1}^{\frac{n-2}{2}} a_{k} f_{2 n+1, k}$ with $a_{k} \in \mathbb{Z}$. Using this formula and the above discussion, we conclude that $\mathrm{H}^{2 n+1}(G, \mathbb{Z}(0)) \subset \mathcal{U}$ also for even $n$.

Next, we claim that the element $\psi_{0} \nu^{n}$ generates $\mathrm{H}^{4 n}\left(G, M^{(a b)}(0)\right)$, the element $\psi_{1} \nu^{n}$ generates $\mathrm{H}^{4 n+2}\left(G, M^{(a b)}(0)\right)$, the element $\varphi_{0} \nu^{n}$ generates $\mathrm{H}^{4 n}\left(G, M^{(b)}(0)\right)$, and the element $\varphi_{1} \nu^{n}$ generates $\mathrm{H}^{4 n+2}\left(G, M^{(b)}(0)\right)$ for any $n \geq 0$. Since $\mathrm{H}^{n}\left(G, M^{(a b)}(0)\right)=$ $\mathrm{H}^{n}\left(G, M^{(b)}(0)\right)=0$ for odd $n$, the inclusion

$$
\mathrm{H}^{*}\left(G, M^{(a b)}(0)\right) \cup \mathrm{H}^{*}\left(G, M^{(b)}(0)\right) \subset \mathcal{T} .
$$

will follow from the above claim. Using induction on $n$ and part 2) of Lemma 5, it is easy to show that the elements $\varphi_{0} \nu^{n}, \varphi_{1} \nu^{n}, \psi_{0} \nu^{n}$, and $\psi_{1} \nu^{n}$ have the last but one coordinate equal to 1 . Then (3.8) and (3.9) imply that $\psi_{0} \nu^{n}$ generates $\mathrm{H}^{4 n}\left(G, M^{(a b)}(0)\right)$, and that $\psi_{1} \nu^{n}$ generates $\mathrm{H}^{4 n+2}\left(G, M^{(a b)}(0)\right)$. Similarly, $\varphi_{0} \nu^{n}$ generates $\mathrm{H}^{4 n}\left(G, M^{(b)}(0)\right)$ and $\varphi_{1} \nu^{n}$ generates $\mathrm{H}^{4 n+2}\left(G, M^{(b)}(0)\right)$.

It remains to prove that $\mathrm{H}^{*}\left(G, M^{(a)}(0)\right) \subset \mathcal{T}$. First, we prove that $\mathrm{H}^{2 n}\left(G, M^{(a)}(0)\right) \subset$ $\mathcal{T}$ for $n \geq 0$. The proof of Lemma 4 shows that $\theta_{0}$ generates $\mathrm{H}^{0}\left(G, M^{(a)}(0)\right)$. Then we 
use induction on $n$. Assume that $n>0$ and $\mathrm{H}^{2(n-1)}\left(G, M^{(a)}(0)\right) \subset \mathcal{T}$. Then (5.2) implies that the elements $v_{1} f_{2 n-2,2 k}^{(a)}=f_{2 n, 2 k}^{(a)}, 0 \leq k \leq \frac{n-1}{2}$, and $v_{1} g_{2 n-2,2 k}^{(a)}=g_{2 n, 2 k}^{(a)}$, $0 \leq k \leq \frac{n-2}{2}$, of $\mathrm{H}^{2 n}\left(G, M^{(a)}(0)\right)$ lie in $\mathcal{T}$. Using part 2) of Lemma 5 , we check by induction on $k$ that $\theta_{0} \nu^{k}$ and $\theta_{1} \nu^{k}$ can be presented in $\mathbb{Z}^{8 k+2}$ and in $\mathbb{Z}^{8 k+6}$, respectively, by vectors with the last but one coordinate equal to 1 . The proof of Lemma 4 shows that, in $\mathrm{H}^{2 n}\left(G, M^{(a)}(0)\right)$, we have

$$
\theta_{0} \nu^{\frac{n}{2}}=-f_{2 n, n}^{(a)}+\sum_{k=0}^{\frac{n-2}{2}}\left(a_{k} f_{2 n, 2 k}^{(a)}+a_{k}^{\prime} g_{2 n, 2 k}^{(a)}\right)
$$

if $n: 2$, and

$$
\theta_{1} \nu^{\frac{n-1}{2}}=g_{2 n, n-1}^{(a)}+\sum_{k=0}^{\frac{n-3}{2}}\left(a_{k} f_{2 n, 2 k}^{(a)}+a_{k}^{\prime} g_{2 n, 2 k}^{(a)}\right)+a_{\frac{n-1}{2}} f_{2 n, n-1}^{(a)}
$$

if $n \% 2$ (in the two identities, all $a_{k}, a_{k}^{\prime}$ are integers). Consequently, $\mathrm{H}^{2 n}\left(G, M^{(a)}(0)\right) \subset \mathcal{T}$. Next, we check that $\mathrm{H}^{2 n+1}\left(G, M^{(a)}(0)\right) \subset \mathcal{T}$ for $n \geq 0$. The proof of Lemma 4 implies that $\mathrm{H}^{1}\left(G, M^{(a)}(0)\right)=0$ and that $\rho_{1}$ generates $\mathrm{H}^{3}\left(G, M^{(a)}(0)\right)$. Then we apply induction on $n$. Assume that $n>1$ and $\mathrm{H}^{2 n-1}\left(G, M^{(a)}(0)\right) \subset \mathcal{T}$. Then, by (5.2), the elements $v_{1} f_{2 n-1, k}^{(a)}=f_{2 n+1, k}^{(a)}$ of $\mathrm{H}^{2 n+1}\left(G, M^{(a)}(0)\right)(0 \leq k \leq n-1)$ lie in $\mathcal{T}$. Then, using induction on $k$, part 2) of Lemma 5, and the fact that $2 f_{2 n+1, n}^{(a)} \in \operatorname{Im} \delta^{*}\left(M^{(a)}\right)$, we see that $\rho_{1} \nu^{k}$, $\rho_{2} \nu^{k}$ can be presented in $\mathbb{Z}^{8 k+8}$ and in $\mathbb{Z}^{8 k+12}$, respectively, by vectors of the form $\left(v, \frac{r}{2}, \mathbb{O}_{3}\right)^{\mathrm{T}}$, where $v$ is a row of suitable length. Then the proof of Lemma 4 shows that

$$
\rho^{\prime}=f_{2 n+1, n}^{(a)}+\sum_{k=1}^{n-1} a_{k} f_{2 n+1, k}^{(a)}
$$

in $\mathrm{H}^{2 n+1}\left(G, M^{(a)}(0)\right)$, where $a_{k} \in \mathbb{Z}(1 \leq k \leq n-1)$, and moreover, $\rho^{\prime}=\rho_{1} \nu^{\frac{n-1}{2}}$ for $n$ odd, and $\rho^{\prime}=\rho_{2} \nu^{\frac{n-2}{2}}$ for $n$ even. Thus, $f_{2 n+1, n}^{(a)} \in \mathcal{T}$, which implies that $\mathrm{H}^{2 n+1}\left(G, M^{(a)}(0)\right) \subset \mathcal{T}$. This completes the proof of the inclusion $\mathrm{H}^{*}(G, \widetilde{R}) \subset \mathcal{T}$.

Corollary 4. The elements $v_{1}, v_{2}, \nu$, and $\omega$ generate a subalgebra of $\mathrm{H}^{*}(G, \widetilde{R})$ isomorphic to the cohomology algebra of the group $G$.

\section{$\S 6$. Relations}

This section is devoted to deriving relations among the elements of the set $\mathcal{X}$ (see (5.1)).

Lemma 6. 1) For $v_{2}$, we can find translates with numbers $0,1,2$ so that the following identities are fulfilled:

$$
\begin{aligned}
& T^{0}\left(v_{2}\right)=\left(\begin{array}{lll}
0 & -1 & 2
\end{array}\right), \\
& T^{1}\left(v_{2}\right)=\left(\begin{array}{cccc}
0 & a L a & 2 a L a & 0 \\
0 & L \sum_{i=0}^{r-1}(r-i) b^{i(r+1)} & \left(b^{r}-L\right)(a L a-1) & 2
\end{array}\right), \\
& T^{2}\left(v_{2}\right)=\left(\begin{array}{cccc}
0 \star & 2(L a)^{2} & 0 & 0 \\
0 \star & \left(b^{r}-L\right)(a L a-1) & 2 a L a & 0 \\
0 \star & \frac{r(r+1)^{2}}{2}-r(r+1) a & -1 & 2
\end{array}\right),
\end{aligned}
$$

where $\left(T^{2}\left(v_{2}\right)\right)_{* 2}$ denotes a suitable column.

2) For $\omega$, we can find translates with numbers 0, 1, 2, 3 so that the following identities are fulfilled:

$$
T^{0}(\omega)\left(e_{1}^{5}\right)=T^{0}(\omega)\left(e_{2}^{5}\right)=T^{0}(\omega)\left(e_{3}^{5}\right)=0, \quad T^{0}(\omega)\left(e_{4}^{5}\right)=-x_{2}, \quad T^{0}(\omega)\left(e_{5}^{5}\right)=r ;
$$




$$
\begin{aligned}
& T^{1}(\omega)\left(e_{1}^{6}\right)=T^{1}(\omega)\left(e_{2}^{6}\right)=T^{1}(\omega)\left(e_{3}^{6}\right)=0 \\
& T^{1}(\omega)\left(e_{4}^{6}\right)=x_{2}\left(\begin{array}{c}
t^{2} \\
S_{1} a
\end{array}\right), \quad T^{1}(\omega)\left(e_{5}^{6}\right)=r\left(\begin{array}{c}
t^{2} \\
S_{1} a
\end{array}\right)-x_{2}\left(\begin{array}{c}
0 \\
S_{2}
\end{array}\right) \\
& T^{2}(\omega)\left(e_{1}^{7}\right)=T^{2}(\omega)\left(e_{2}^{7}\right)=T^{2}(\omega)\left(e_{3}^{7}\right)=0, \quad T^{2}(\omega)\left(e_{4}^{7}\right)=-x_{2}\left(\begin{array}{c}
t^{4} \\
t^{2} S_{1} a \\
\frac{r}{4} y_{2}^{2}
\end{array}\right), \\
& T^{2}(\omega)\left(e_{5}^{7}\right)=r\left(\begin{array}{c}
t^{4} \\
t^{2} S_{1} a \\
\frac{r}{4} y_{2}^{2}
\end{array}\right)+x_{2}\left(\begin{array}{c}
0 \\
t^{2} S_{2} \\
\frac{r}{2} t^{2} a
\end{array}\right) ; \\
& T^{3}(\omega)\left(e_{1}^{8}\right)=T^{3}(\omega)\left(e_{2}^{8}\right)=T^{3}(\omega)\left(e_{3}^{8}\right)=0, \quad T^{3}(\omega)\left(e_{4}^{8}\right)=x_{2}\left(\begin{array}{c}
t^{4} S_{1} a \\
\frac{r}{4} y_{2}^{2} t^{2} \\
S_{3} a
\end{array}\right)
\end{aligned}
$$

where $S_{1}$ and $S_{2}$ are elements in $R$ representable as integral linear combinations of powers of $b$ and satisfying

$$
(b-1) S_{1}=L^{2}-t^{2}, \quad(b-1) S_{2}=N_{b}-2 r \quad \text { and } \varepsilon\left(S_{1}\right)=\varepsilon\left(S_{2}\right)=r
$$

moreover, $S_{3} \in R$ satisfies

$$
(b-1) S_{3}=\frac{y_{2} t^{4}}{2} S_{1}-\frac{r y_{2}^{2} t^{2}}{4} L+\frac{r y_{2}^{2}}{4} L^{2}-\frac{x_{2} y_{2} r t^{2}}{2} .
$$

3) For $\theta_{1}$, we can find translates with numbers $0,1,2$ so that the following identities are fulfilled:

$$
\begin{aligned}
T^{0}\left(\theta_{1}\right) & =\left(\begin{array}{ccc}
0 & 0 & 1 \\
1 & -1 & 0
\end{array}\right), \\
T^{1}\left(\theta_{1}\right) & =\left(\begin{array}{cccc}
0 & \frac{r}{2} & a L a & 0 \\
1 & 1 & 0 & 0 \\
0 & \sum_{i=1}^{\frac{r}{2}} \sum_{j=0}^{2 i-1} b^{j} a+1 & -b^{r} \sum_{i=1}^{\frac{r}{2}} b^{2 i-1} & 1 \\
0 & -\sum_{i=1}^{\frac{r}{2}} b^{2 i-1} a-1 & \sum_{i=1}^{r} b^{2 i-1} & 0
\end{array}\right), \\
T^{2}\left(\theta_{1}\right)= & \left(\begin{array}{cccc}
0 \star & (L a)^{2} & 0 & 0 \\
1 \star & 0 & 0 & 0 \\
0 \star & \sum_{i=1}^{\frac{r}{2}} b^{2 i-1} & a L a & 0 \\
0 \star & -\sum_{i=1}^{r} b^{2 i-1} & 0 & 0 \\
0 \star & \frac{r^{2}}{4} a+1 & -1 & 1 \\
0 \star & \frac{r}{2} a-1 & 1 & 0
\end{array}\right),
\end{aligned}
$$

where $\left(T^{2}\left(\theta_{1}\right)\right)_{* 2}$ denotes a suitable column.

4) For $\varphi_{1}$, we can find translates such that, for $k \geq 0$, they are determined by matrices of the form

$$
T^{k}\left(\varphi_{1}\right)=\left(\mathbb{O}^{2 k+2} \quad A_{k}^{\prime}[1, r]_{2 k+1}^{2 k+2}\right),
$$

where $A_{k}^{\prime}$ is a matrix of size $(2 k+2) \times(k+1)$.

5) For $\psi_{1}$, we can find translates such that, for $k \geq 0$, they are determined by matrices of the form

$$
T^{k}\left(\psi_{1}\right)=\left(\mathbb{O}^{2 k+2} \quad A_{k}^{\prime \prime}[1,0]_{2 k+1}^{2 k+2}\right),
$$

where $A_{k}^{\prime \prime}$ is a matrix of size $(2 k+2) \times(k+1)$.

Remark 2. If $M$ is one of the modules $M^{(b)}, M^{(a b)}, M^{(a)}$, and $\left\{m_{1}, m_{2}\right\}$ is the standard $\mathbb{Z}$-basis of $M$ (see (3.4)) then, in the description of matrices for translates of $\theta_{1}, \varphi_{1}, \psi_{1}$, for the modules $Q_{n} \otimes M$, we use the sets of generators of the form $e_{i}^{n} \otimes m_{j}(1 \leq i \leq$ $n+1, j \in\{1,2\})$. 
Proof of Lemma 6, 1) We must prove that $\varepsilon T^{0}\left(v_{2}\right)=v_{2}, d_{0} T^{1}\left(v_{2}\right)=T^{0}\left(v_{2}\right) d_{2}$, and $d_{1} T^{2}\left(v_{2}\right)=T^{1}\left(v_{2}\right) d_{3}$. The first relation is obvious, and for verifying the last relation it suffices to consider only the elements $e_{1}^{4}, e_{3}^{4}, e_{4}^{4}, e_{5}^{4}$. Observe that

$$
\begin{aligned}
(b-1) L & \sum_{i=0}^{r-1}(r-i) b^{i(r+1)}=\left(b^{r+1}-1\right) \sum_{i=0}^{r-1}(r-i) b^{i(r+1)} \\
& =\sum_{i=1}^{r}(r-i+1) b^{i(r+1)}-\sum_{i=0}^{r-1}(r-i) b^{i(r+1)}=\sum_{i=1}^{r} b^{i(r+1)}-r=a L a-(r+1)
\end{aligned}
$$

and

$$
\begin{aligned}
& (b-1)\left(b^{r}-L\right)(a L a-1)=\left(1-b^{r}\right)(a L a-1)=\left(1-b^{r}\right) \sum_{i=1}^{r} b^{i(r+1)} \\
& =\sum_{i=1}^{r} b^{i(r+1)}-\sum_{i=1}^{r} b^{(i+r)(r+1)}=2 \sum_{i=0}^{r} b^{i(r+1)}-2-\sum_{i=1}^{r r} b^{(i+r)(r+1)}=2 a L a-2-N_{b} .
\end{aligned}
$$

These identities imply $d_{0} T^{1}\left(v_{2}\right)=T^{0}\left(v_{2}\right) d_{2}$. Next, it is easily seen that the maps $d_{1} T^{2}\left(v_{2}\right)$ and $T^{1}\left(v_{2}\right) d_{3}$ coincide at $e_{1}^{4}, e_{4}^{4}, e_{5}^{4}$. To check that they coincide at $e_{3}^{4}$, we need to prove the identities

$$
\begin{aligned}
2(a+1)(L a)^{2}+2 a L a-2-N_{b} & =a L a N_{b}+2 a L a(L a+1), \\
(1-L a)\left(b^{r}-L\right)(a L a-1)+ & \left(\frac{r(r+1)^{2}}{2}-r(r+1) a\right) N_{b} \\
& =L \sum_{i=0}^{r-1}(r-i) b^{i(r+1)} N_{b}+\left(b^{r}-L\right)(a L a-1)(L a+1) .
\end{aligned}
$$

Since $b^{i} N_{b}=N_{b}$ for any $i \in \mathbb{Z}$, the first identity is equivalent to

$$
(L a)^{2}=\frac{r+2}{2} N_{b}+1,
$$

and the second is equivalent to

$$
\left((a L)^{2}+(L a)^{2}-\left(b^{r}+1\right) L-\left(b^{r}+1\right) a L a+2 b^{r}\right) L a=r(r+1) N_{b} a .
$$

Since $\left(b^{r}+1\right) L=\left(b^{r}+1\right) a L a=N_{b}+b^{r}+1$, the last identity can be written in the form $\left((a L)^{2}+(L a)^{2}-2\right) L a=(r+2)(r+1) N_{b} a$. Because of $(a L)^{2}=a(L a)^{2} a$, it remains to verify (6.1). Observe that

$$
(b-1)(L a)^{2}=(b-1) L a L a=\left(b^{r+1}-1\right) \sum_{i=0}^{r} b^{i(r+1)}=b^{(r+1)^{2}}-1=b-1 .
$$

Since $(L a)^{2}$ is an integral linear combination of powers of $b$, we have $(L a)^{2}=\varkappa N_{b}+1$ for some $\varkappa \in \mathbb{Z}$. Finally, we observe that $\varepsilon\left((L a)^{2}\right)=(r+1)^{2}$, i.e., $\varkappa=\frac{(r+1)^{2}-1}{2 r}=\frac{r+2}{2}$.

2) First, we prove the existence of elements $S_{1}, S_{2}, S_{3}$ with the required properties. Observe that the element $S_{1}(\gamma)=\sum_{i=0}^{r-1}(r-i) b^{i}(L+t)-\gamma N_{b}$ satisfies the equation $(b-1) S_{1}(\gamma)=L^{2}-t^{2}$ for any integer $\gamma$. It remains to show that $\varepsilon\left(\sum_{i=0}^{r-1}(r-i) b^{i}(L+t)\right)$ has remainder $r$ under division by $2 r$ : indeed, we have $\varepsilon\left(\sum_{i=0}^{r-1}(r-i) b^{i}(L+t)\right)=$ $\frac{r(r+1)}{2} \cdot(2 r+2) \equiv r \bmod 2 r$. Similarly, the element $S_{2}$ is chosen in the form $S_{2}=$ $\sum_{i=0}^{2 r-1}(2 r-i) b^{i}-\gamma N_{b}$ (for suitable $\left.\gamma \in \mathbb{Z}\right)$. Since

$$
\left(\frac{y_{2} t^{4}}{2} S_{1}-\frac{r y_{2}^{2} t^{2}}{4} L+\frac{r y_{2}^{2}}{4} L^{2}-\frac{x_{2} y_{2} r t^{2}}{2}\right) N_{b}=\left(\frac{y_{2} t^{4} r}{2}-\frac{r y_{2}^{2} t^{3}}{4}+\frac{r y_{2}^{2} t^{2}}{4}-\frac{x_{2} y_{2} r t^{2}}{2}\right) N_{b}=0
$$


we see that an element $S_{3}$ with the required properties also exists.

Next, we prove the identities $\varepsilon T^{0}(\omega)=\omega$ and $d_{i} T^{i+1}(\omega)\left(e_{j}^{i+6}\right)=T^{i}(\omega) d_{i+5}\left(e_{j}^{i+6}\right)$ for the pairs $(i, j)$ such that either $i \in\{0,1\}, 1 \leq j \leq 5$, or $i=2,1 \leq j \leq 4$. The first identity is verified without difficulties. The second is obvious for $1 \leq j \leq 3$. It is easily seen that $d_{0} T^{1}(\omega)\left(e_{j}^{6}\right)=T^{0}(\omega) d_{5}\left(e_{j}^{6}\right)$ for $j \in\{4,5\}$. We can establish by direct calculations that

$$
\begin{aligned}
& d_{1} T^{2}(\omega)\left(e_{4}^{7}\right)-T^{1}(\omega) d_{6}\left(e_{4}^{7}\right)=\left(\begin{array}{c}
0 \\
x_{2}\left(-\frac{r}{4} y_{2}^{2} N_{b}+Y_{1}\right)
\end{array}\right) \\
& d_{1} T^{2}(\omega)\left(e_{5}^{7}\right)-T^{1}(\omega) d_{6}\left(e_{5}^{7}\right)=\left(\begin{array}{c}
0 \\
r\left(\frac{r}{4} y_{2}^{2} N_{b}-Y_{1}\right)+x_{2}\left(\frac{r}{2}\left(t^{2}-2\right) N_{b}-Y_{2}\right) a
\end{array}\right)
\end{aligned}
$$

and

$$
d_{2} T^{3}(\omega)\left(e_{4}^{7}\right)-T^{2}(\omega) d_{7}\left(e_{4}^{7}\right)=\left(\begin{array}{c}
0 \\
x_{2} t^{2}\left(\frac{r}{4} y_{2}^{2} N_{b}-Y_{1}\right) \\
0
\end{array}\right)
$$

where $Y_{1}=S_{1} a L^{2} a+t^{2} L a S_{1} a-x_{2} y_{2} S_{2}$ and $Y_{2}=-2 r S_{1}-S_{2} L^{2}+t^{2} L a S_{2} a$. Since $(L a)^{2}=\frac{r+2}{2} N_{b}+1$, we can easily show that $(b-1) Y_{1}=(b-1) Y_{2}=0, \varepsilon\left(Y_{1}\right)=\frac{r^{2}}{2} y_{2}^{2}$, and $\varepsilon\left(Y_{2}\right)=r^{2}\left(t^{2}-2\right)$, and the desired identities follow.

3) We must prove that $\left(\varepsilon \otimes \operatorname{id}_{M^{(a)}(0)}\right) T^{0}\left(\theta_{1}\right)=\theta_{1},\left(d_{0} \otimes \operatorname{id}_{M^{(a)}(0)}\right) T^{1}\left(\theta_{1}\right)=T^{0}\left(\theta_{1}\right) d_{2}$, and $\left(d_{1} \otimes \mathrm{id}_{M^{(a)}(0)}\right) T^{2}\left(\theta_{1}\right)=T^{1}\left(\theta_{1}\right) d_{3}$. Moreover, it suffices to check the last identity only at the elements $e_{1}^{4}, e_{3}^{4}, e_{4}^{4}, e_{5}^{4}$. All these identities can be verified by direct, although cumbersome, calculations using formula (6.1) and the identities

$$
b^{j} \sum_{i=1}^{r} b^{2 i}=b^{j+1} \sum_{i=1}^{r} b^{2 i-1}= \begin{cases}\sum_{i=1}^{r} b^{2 i} & \text { if } j: 2, \\ \sum_{i=1}^{r} b^{2 i-1} & \text { if } j \% 2 .\end{cases}
$$

These verifications are left to the reader.

The two remaining parts of the lemma are established without difficulties.

Proposition 3. In the algebra $\mathrm{H}^{*}(G, \widetilde{R})$, the following relations are fulfilled:

$$
\begin{gathered}
2 v_{1}=r v_{2}=2 r \nu=2 \omega=2 r \varphi_{1}=r \theta_{0} \nu=r \theta_{1}=2 \rho_{1}=2 \rho_{2}=2 r \psi_{1}=0 ; \\
v_{1} v_{2}=0, \quad v_{2}^{2}=4 \nu, \quad v_{2} \omega=0, \quad \omega^{2}=0, \quad \lambda^{r}=1 ; \\
\psi_{0}^{2}=2\left(\lambda+\lambda^{\frac{r+2}{2}}\right), \quad \psi_{0} \psi_{1}=\left(\lambda+\lambda^{\frac{r+2}{2}}\right) v_{2}, \quad \psi_{1}^{2}=\psi_{0}^{2} \nu ; \\
\psi_{0} v_{1}=r \psi_{1}, \quad \psi_{1} v_{1}=r \psi_{0} \nu, \quad \psi_{0} v_{2}=(2+r) \psi_{1}, \quad \psi_{1} v_{2}=(2+r) \psi_{0} \nu, \\
\psi_{0} \omega=\psi_{1} \omega=0, \quad \psi_{0} \lambda^{\frac{r}{2}}=\psi_{0}, \quad \psi_{1} \lambda^{\frac{r}{2}}=(1+r) \psi_{1} ; \\
\theta_{0}^{2}=2\left(1+\lambda^{\frac{r}{2}}\right), \quad \theta_{0} \theta_{1}=\left(1+\lambda^{\frac{r}{2}}\right)\left(v_{1}+v_{2}\right), \quad \theta_{1}^{2}=v_{1}^{2}+\theta_{0}^{2} \nu, \quad \theta_{0} \rho_{1}=0, \\
\theta_{1} \rho_{1}=\theta_{0} \rho_{2}=\left(1+\lambda^{\frac{r}{2}} \omega, \quad \theta_{1} \rho_{2}=v_{1} \omega, \quad \rho_{1}^{2}=\rho_{1} \rho_{2}=\rho_{2}^{2}=0 ;\right. \\
\theta_{0} v_{2}=2 \theta_{1}, \quad \theta_{1} v_{2}=2 \theta_{0} \nu, \quad \theta_{0} \omega=\rho_{1} v_{1}, \quad \theta_{1} \omega=\rho_{2} v_{1}, \\
\rho_{1} v_{2}=\rho_{2} v_{2}=\rho_{1} \omega=\rho_{2} \omega=0, \\
\theta_{0} \lambda^{\frac{r}{2}}=\theta_{0}, \quad \theta_{1} \lambda^{\frac{r}{2}}=\theta_{1}+\theta_{0} v_{1}, \rho_{1} \lambda^{\frac{r}{2}}=\rho_{1}, \quad \rho_{2} \lambda^{\frac{r}{2}}=\rho_{2}+\rho_{1} v_{1} ; \\
\varphi_{0}^{2}=2\left(\lambda+\lambda^{\frac{r+2}{2}}\right), \quad \varphi_{0} \varphi_{1}=\left(1+\frac{r}{2}\right)\left(\lambda+\lambda^{\frac{r+2}{2}}\right) v_{2}, \quad \varphi_{1}^{2}=\varphi_{0}^{2} \nu ; \\
\varphi_{0} v_{1}=\varphi_{1} v_{1}=\varphi_{0} \omega=\varphi_{1} \omega=0, \quad \varphi_{0} v_{2}=2 \varphi_{1}, \quad \varphi_{1} v_{2}=2 \varphi_{0} \nu \\
\varphi_{0} \lambda^{\frac{r}{2}}=\varphi_{0}, \quad \varphi_{1} \lambda^{\frac{r}{2}}=(1+r) \varphi_{1} ;
\end{gathered}
$$




$$
\begin{aligned}
\psi_{0} \theta_{0} & =2 \varphi_{0}, \psi_{0} \theta_{1}=\psi_{1} \theta_{0}=2 \varphi_{1}, \psi_{1} \theta_{1}=2 \varphi_{0} \nu \\
\psi_{0} \rho_{1} & =\psi_{0} \rho_{2}=\psi_{1} \rho_{1}=\psi_{1} \rho_{2}=0 \\
\varphi_{0} \psi_{0} & =2 \theta_{0} \lambda, \varphi_{0} \psi_{1}=\varphi_{1} \psi_{0}=2 \theta_{1} \lambda, \varphi_{1} \psi_{1}=2 \theta_{0} \nu \lambda \\
\varphi_{0} \theta_{0} & =2 \psi_{0}, \varphi_{1} \theta_{0}=2 \psi_{1}, \varphi_{0} \theta_{1}=(2+r) \psi_{1}, \varphi_{1} \theta_{1}=2 \psi_{0} \nu \\
\varphi_{0} \rho_{1} & =\varphi_{0} \rho_{2}=\varphi_{1} \rho_{1}=\varphi_{1} \rho_{2}=0 .
\end{aligned}
$$

Proof. We have $r \theta_{0} \nu=0$, because $\theta_{0} \nu$ is an element of the group $\mathrm{H}^{4}\left(G, M^{(a)}(0)\right)$, and $\mathrm{H}^{4}\left(G, M^{(a)}(0)\right)=\mathbb{Z}_{2}^{2} \oplus \mathbb{Z}_{r}$ by Lemma 4. The remaining identities in the first line follow from the definition of the elements of the set $\mathcal{X}$ (see (5.1)) and the proofs of Lemmas 24,

Then (5.2) and (3.6) imply that $v_{1} v_{2}=v_{1} f_{2,1}=f_{4,1}=0$ in $\mathrm{H}^{4}(G, \widetilde{R})$. From part 1) of Lemma 6. it follows that $v_{2}^{2} \in \mathbb{Z}^{5}$ has the first coordinate equal to 0 and the last equal to 4. Now, from (3.6) and the definition of $\nu$ it follows that $v_{2}^{2}=4 \nu$. Since $v_{2} \omega \in \mathrm{H}^{7}(G, \mathbb{Z}(0)) \simeq \mathbb{Z}_{2}$ and $v_{1} \omega=f_{7,3}$ is a generator of this group (see the proof of Lemma 21), we have $v_{2} \omega=\varkappa v_{1} \omega$ for some $\varkappa \in \mathbb{Z}$. Multiplying this by $v_{1}$, we obtain $\varkappa v_{1}^{2} \omega=0$. We have $v_{1}^{2} \omega=f_{9,4} \neq 0$ in $\mathrm{H}^{9}(G, \mathbb{Z}(0))$, which implies that $\varkappa \vdots 2$, i.e., $v_{2} \omega=0$. Furthermore, since $\lambda^{k}$ takes 1 to $b^{2 k}$, we see that $\lambda^{r}=1$.

Since $T^{0}\left(\psi_{0}\right)$ takes 1 to $1 \otimes\left(a b+a b^{t}\right)$, the element $\psi_{0}^{2}$ takes 1 to $\left(a b+a b^{t}\right)^{2}=2\left(b^{2}+\right.$ $\left.b^{r+2}\right)$, i.e., $\psi_{0}^{2}=2\left(\lambda+\lambda^{\frac{r+2}{2}}\right)$. Since $\mu\left(\psi_{0} \otimes \mathrm{id}\right) T^{0}\left(\psi_{1}\right)\left(e_{1}^{2}\right)=0, \mu\left(\psi_{0} \otimes \mathrm{id}\right) T^{0}\left(\psi_{1}\right)\left(e_{2}^{2}\right)=$ $-a b\left(a b+a b^{t}\right)=-\left(b^{2}+b^{r+2}\right)$, and $\mu\left(\psi_{0} \otimes \mathrm{id}\right) T^{0}\left(\psi_{1}\right)\left(e_{3}^{2}\right)=\left(a b+a b^{t}\right)^{2}=2\left(b^{2}+b^{r+2}\right)$, we obtain $\psi_{0} \psi_{1}=\left(\lambda+\lambda^{\frac{r+2}{2}}\right) v_{2}$. Using part 3) of Lemma 6, we easily check that $\psi_{1}^{2}$ is represented in $\mathrm{H}^{4}(G, \widetilde{R})$ by an element that takes $e_{1}^{4}$ to 0 and $e_{5}^{4}$ to $2\left(b^{2}+b^{r+2}\right)$. Then (3.6) Shows that, as an element of $\mathrm{H}^{4}(G, \mathbb{Z}(1)) \oplus \mathrm{H}^{4}\left(G, \mathbb{Z}\left(\frac{r+2}{2}\right)\right), \psi_{1}^{2}$ is represented in the form $\left(2 f_{4,2}, 2 f_{4,2}\right)$, whence $\psi_{1}^{2}=2\left(\lambda+\lambda^{\frac{r+2}{2}}\right) \nu=\varphi_{0}^{2} \nu$.

Using (3.9), we obtain the following relations in $\mathrm{H}^{*}\left(G, M^{(a b)}(0)\right)$ :

$$
\begin{aligned}
& \psi_{0} v_{1}=[1]_{1}^{6}=f_{2,0}^{(a b)}=r f_{2,1}^{(a b)}=r \psi_{1}, \\
& \psi_{1} v_{1}=f_{4,1}^{(a b)}=r f_{4,2}^{(a b)}=r \psi_{0} \nu
\end{aligned}
$$

(earlier we have proved that $\left\langle\psi_{0} \nu\right\rangle=\mathrm{H}^{4}\left(G, M^{(a b)}(0)\right)$ ), and

$$
\psi_{0} T^{0}\left(v_{2}\right)=2 f_{2,1}^{(a b)}-g_{2,1}^{(a b)}=(2+r) f_{2,1}^{(a b)}=(2+r) \psi_{1} .
$$

Using part 1) of Lemma 6 and the fact that $L$ acts on $M^{(a b)}=\mathbb{Z}^{2}$ as the matrix $\left(\begin{array}{cc}r+1 & \frac{r}{2} \\ 0 & 1\end{array}\right)$, we conclude that $\psi_{1} T^{2}\left(v_{2}\right)$ is presented in $\mathbb{Z}^{10}$ by a vector of the form $\left(v^{\prime}, \frac{r\left(r^{2}+r-1\right)}{2}, 0,-(r+1),-2,2,0\right)^{\mathrm{T}}$, where $v^{\prime}$ is a row of length 4 . Then (3.8) and (3.9) imply the following identities in $\mathrm{H}^{4}\left(G, M^{(a b)}\right)$ :

$$
\psi_{1} v_{2}=\frac{r\left(r^{2}+r-1\right)}{2} f_{4,1}^{(a b)}-(r+1) g_{4,2}^{(a b)}+2 f_{4,2}^{(a b)}=(2+r) f_{4,2}^{(a b)}=(2+r) \psi_{0} \nu .
$$

Next, we have $\psi_{0} \omega=\psi_{1} \omega=0$, because $\mathrm{H}^{5}\left(G, M^{(a b)}\right)=\mathrm{H}^{7}\left(G, M^{(a b)}\right)=0$. Since $\psi_{0}$ takes $e_{1}^{0}$ to $a b+a b^{t}$, we see that $\psi_{0} \lambda^{\frac{r}{2}}$ also takes $e_{1}^{0}$ to $a b+a b^{t}$, whence $\psi_{0} \lambda^{\frac{r}{2}}=\psi_{0}$. Since $\psi_{1}$ takes $e_{1}^{2}, e_{2}^{2}$, and $e_{3}^{2}$ to 0 , to $-a b$, and to $a b+a b^{t}$, respectively, $\psi_{1} \lambda^{\frac{r}{2}}$ takes the same elements to 0 , to $-a b^{t}=a b-\left(a b+a b^{t}\right)$, and to $a b+a b^{t}$, respectively, and hence, in $\mathrm{H}^{2}\left(G, M^{(a b)}\right)$ we have $\psi_{1} \lambda^{\frac{r}{2}}=f_{2,1}^{(a b)}-g_{2,1}^{(a b)}=(r+1) f_{2,1}^{(a b)}=(r+1) \psi_{1}$.

The relations for $\theta_{0}^{2}$ and $\theta_{0} \theta_{1}$ are established much as those for $\psi_{0}^{2}$ and $\psi_{0} \psi_{1}$. Using part 4) of Lemma 6. we show that $\theta_{1}^{2}$ can be presented in $\mathrm{H}^{4}(G, \widetilde{R})$ by an element that takes $e_{1}^{4}$ to 1 and $e_{5}^{4}$ to $2\left(1+b^{r}\right)$. From (3.6), it follows that, as an element of $\mathrm{H}^{4}(G, \mathbb{Z}(0)) \oplus \mathrm{H}^{4}\left(G, \mathbb{Z}\left(\frac{r}{2}\right)\right), \theta_{1}^{2}$ is presented in the form $\left(f_{4,1}+2 f_{4,2}, 2 f_{4,2}\right)$, whence $\theta_{1}^{2}=v_{1}^{2}+2\left(1+\lambda^{\frac{r}{2}}\right) \nu=v_{1}^{2}+\theta_{0}^{2} \nu$. 
We have $\theta_{0} \rho_{1}=0$, because $\mathrm{H}^{3}\left(G, \mathbb{Z}\left(\frac{r}{2}\right)\right)=\mathrm{H}^{3}(G, \mathbb{Z}(0))=0$. Next, it is easily seen that $\theta_{0} \rho_{2}=\left(1+\lambda^{\frac{r}{2}}\right) \omega$ in $\mathrm{H}^{5}(G, \mathbb{Z}(0)) \oplus \mathrm{H}^{5}\left(G, \mathbb{Z}\left(\frac{r}{2}\right)\right)$. Now, we observe immediately that $\lambda^{\frac{r}{2}} \theta_{0}=\theta_{0}$,

$$
\lambda^{\frac{r}{2}} \theta_{1}=f_{2,0}^{(a)}-f_{2,1}^{(a)}-g_{2,0}^{(a)}=f_{2,0}^{(a)}+g_{2,0}^{(a)}=\theta_{0} v_{1}+\theta_{1},
$$

$\lambda^{\frac{r}{2}} \rho_{1}=\rho_{1}-x_{1} g_{3,1}^{(a)}=\rho_{1}$, and

$$
\lambda^{\frac{r}{2}} \rho_{2}=\rho_{2}-x_{2} g_{5,2}^{(a)}=\rho_{2}+f_{5,1}^{(a)}=\rho_{2}+v_{1} \rho_{1} .
$$

The proof of Proposition 2 shows that $\theta_{1} \rho_{1}=\left(\varkappa_{1}+\varkappa_{2} \lambda^{\frac{r}{2}}\right) \omega$ for some $\varkappa_{1}, \varkappa_{2} \in \mathbb{Z}$. Multiplying this by $\lambda^{\frac{r}{2}}$, we obtain $\varkappa_{1} \equiv \varkappa_{2}(\bmod 2)$, and hence, $\theta_{1} \rho_{1}=\varkappa_{1}\left(1+\lambda^{\frac{r}{2}}\right) \omega$. Since $2 \omega=0$, we may assume that $\varkappa_{1}$ is equal to 0 or 1 . But $\theta_{1}^{2} \rho_{1}=\left(v_{1}^{2}+\theta_{0}^{2} \nu\right) \rho_{1}=$ $v_{1}^{2} \rho_{1} \neq 0$, whence $\varkappa_{1} \neq 0$, and, consequently, $\theta_{1} \rho_{1}=\left(1+\lambda^{\frac{r}{2}}\right) \omega$. Next, we have $\theta_{0} v_{2}=$ $-f_{2,1}^{(a)}=2 g_{2,0}^{(a)}=2 \theta_{1}$ and $\theta_{0} \omega=2 f_{5,2}^{(a)}-x_{2} g_{5,2}^{(a)}=f_{5,1}^{(a)}=v_{1} \rho_{1}$. Furthermore, $\theta_{1} v_{2} \in$ $\mathrm{H}^{4}\left(G, M^{(a)}(0)\right)$, so that $\theta_{1} v_{2}=\varkappa_{1} \theta_{0} v_{1}^{2}+\varkappa_{2} \theta_{1} v_{1}+\varkappa_{3} \theta_{0} \nu$ for some $\varkappa_{1}, \varkappa_{2}, \varkappa_{3} \in \mathbb{Z}$. Multiplying this by $v_{1}$, we see that $\varkappa_{1} \equiv \varkappa_{2} \equiv 0(\bmod 2)$, whence $\theta_{1} v_{2}=\varkappa_{3} \theta_{0} \nu$. Multiplying the last relation by $\theta_{0}$, we conclude that $\varkappa_{3} \equiv 2(\bmod r)$, whence we $\theta_{1} v_{2}=$ $2 \theta_{0} \nu$.

Now we check that

$$
\theta_{1} \rho_{2}=v_{1} \omega, \quad \theta_{1} \omega=\rho_{2} v_{1} .
$$

Since $\theta_{1} \rho_{2} \in \mathrm{H}^{7}(G, \mathbb{Z}(0)) \oplus \mathrm{H}^{7}\left(G, \mathbb{Z}\left(\frac{r}{2}\right)\right)$ and $\theta_{1} \omega \in \mathrm{H}^{7}\left(G, M^{(a)}(0)\right)$, there exist integers $\varkappa_{1}, \varkappa_{2}, \alpha_{1}, \alpha_{2}, \alpha_{3}$ with

$$
\theta_{1} \rho_{2}=\left(\varkappa_{1}+\varkappa_{2} \lambda^{\frac{r}{2}}\right) v_{1} \omega, \quad \theta_{1} \omega=\alpha_{1} \rho_{2} v_{1}+\alpha_{2} \rho_{1} v_{1}^{2}+\alpha_{3} \rho_{1} \nu
$$

(see the proof of Proposition 2). Multiplying the first identity by $\lambda^{\frac{r}{2}}$ and the second by $\theta_{0}$ and using identities proved above, we get $\varkappa_{1} \equiv \varkappa_{2}+1(\bmod 2)$ and $\alpha_{1} \equiv 1(\bmod 2)$. Then

$$
v_{1}^{2} \omega=\theta_{1}^{2} \omega=\theta_{1} \rho_{2} v_{1}+\alpha_{2} \theta_{1} \rho_{1} v_{1}^{2}+\alpha_{3} \theta_{1} \rho_{1} \nu=v_{1}^{2} \omega+\left(1+\lambda^{\frac{r}{2}}\right)\left(\left(\varkappa_{2}+\alpha_{2}\right) v_{1}^{2} \omega+\alpha_{3} \nu \omega\right) .
$$

Consequently, we can take $\alpha_{3}=0$ and $\alpha_{2}=\varkappa_{2}$, obtaining $\theta_{1} \rho_{2}=v_{1} \omega+\varkappa\left(1+\lambda^{\frac{r}{2}}\right) v_{1} \omega$ and $\theta_{1} \omega=\rho_{2} v_{1}+\varkappa \rho_{1} v_{1}^{2}$ for some integer $\varkappa$. From part 2) of Lemma [6] it follows that $\theta_{1} T^{2}(\omega)\left(e_{1}^{7}\right)=\theta_{1} T^{2}(\omega)\left(e_{2}^{7}\right)=\theta_{1} T^{2}(\omega)\left(e_{3}^{7}\right)=0$ and

$$
\theta_{1} T^{2}(\omega)\left(e_{4}^{7}\right)=-x_{2}\left(t^{4} a-t^{2} a * S_{1} a+\frac{r}{4} y_{2}^{2}\left(a+a b^{r}\right)\right) .
$$

We shall prove that

$$
a * S_{1} a=2 \beta_{1}\left(a+a b^{r}\right)+\beta_{2} a \quad \text { with } \beta_{1}, \beta_{2} \in \mathbb{Z} .
$$

Then the form of the elements $\rho_{2} v_{1}, \rho_{1} v_{1}^{2}$ and the proof of Lemma4 will show immediately that $\varkappa: 2$, which will prove identities (6.2). Since $S_{1}=\sum_{i=1}^{2 r} \gamma_{i} b^{i}$, where $\gamma_{i} \in \mathbb{Z}$ and $\sum_{i=1}^{2 r} \gamma_{i}=r$, it remains to show that $\sum_{i=1}^{r} \gamma_{2 i}-\sum_{i=1}^{r} \gamma_{2 i-1} \vdots 4$. This follows from the fact that $S_{1}=\sum_{i=0}^{r-1}(r-i) b^{i}(L+t)-\gamma N_{b}$ for some integer $\gamma$, and the sum of the coefficients of the even powers of $b$ in the expansion of $L+t$ in powers of $b$ differs from the sum of the coefficients of odd powers of $b$ by the even number $t+1$.

Now, the proof of Proposition 2 shows that, for any $n$, multiplication by $v_{1}$ gives rise to an injective map from $\mathrm{H}^{2 n-1}\left(G, M^{(a)}(0)\right)$ into $\mathrm{H}^{2 n+1}\left(G, M^{(a)}(0)\right)$. Since also $\rho_{1} v_{2} \in$ $\mathrm{H}^{5}\left(G, M^{(a)}(0)\right), \rho_{2} v_{2} \in \mathrm{H}^{7}\left(G, M^{(a)}(0)\right)$, and $v_{1} v_{2}=0$, we obtain $\rho_{1} v_{2}=\rho_{2} v_{2}=0$.

Now we claim that $\rho_{1}^{2}=0$. Since $\rho_{1}^{2} \in \mathrm{H}^{6}(G, \mathbb{Z}(0)) \oplus \mathrm{H}^{6}\left(G, \mathbb{Z}\left(\frac{r}{2}\right)\right)$, we have

$$
\rho_{1}^{2}=\left(\varkappa_{1}+\varkappa_{1}^{\prime} \lambda^{\frac{r}{2}}\right) v_{1}^{3}+\left(\varkappa_{2}+\varkappa_{2}^{\prime} \lambda^{\frac{r}{2}}\right) v_{1} \nu+\left(\varkappa_{3}+\varkappa_{3}^{\prime} \lambda^{\frac{r}{2}}\right) v_{2} \nu
$$


for some $\varkappa_{1}, \varkappa_{1}^{\prime}, \varkappa_{2}, \varkappa_{2}^{\prime}, \varkappa_{3}, \varkappa_{3}^{\prime} \in \mathbb{Z}$. The translates $T^{k}\left(\rho_{1}\right), k \geq 0$, can be constructed so that $T^{k}\left(\rho_{1}\right)\left(e_{k+4}^{k+3}\right)=0$. Then (3.6) and the proof of Proposition 2 imply $\varkappa_{3}=\varkappa_{3}^{\prime}=0$. It is clear that either $\rho_{1}^{2}=0$ or $v_{1} \rho_{1}^{2} \neq 0$. But $v_{1} \rho_{1}^{2}=\theta_{0} \omega \rho_{1}=0$, and we conclude that $\rho_{1}^{2}=0$.

Now we check that $\rho_{1} \omega=\rho_{2} \omega=\rho_{1} \rho_{2}=\rho_{2}^{2}=\omega^{2}=0$. Since $\rho_{1} \omega \in \mathrm{H}^{8}\left(G, M^{(a)}(0)\right)$, we have

$$
\rho_{1} \omega=\varkappa_{1} v_{1}^{4} \theta_{0}+\varkappa_{2} v_{1}^{2} \nu \theta_{0}+\varkappa_{3} \nu^{2} \theta_{0}+\varkappa_{4} v_{1}^{3} \theta_{1}+\varkappa_{5} v_{1} \nu \theta_{1}
$$

for some integers $\varkappa_{i}(1 \leq i \leq 5)$. Multiplying this by $\lambda^{\frac{r}{2}}$, we obtain $\varkappa_{4} \equiv \varkappa_{5} \equiv 0(\bmod 2)$. Multiplying the resulting identity by $\theta_{0}$, we conclude that $\varkappa_{3} \equiv 0(\bmod r)$, whence $\rho_{1} \omega=\varkappa_{1} v_{1}^{4} \theta_{0}+\varkappa_{2} v_{1}^{2} \nu \theta_{0}$. Observe that $v_{1}^{4} \theta_{0}=f_{8,0}^{(a)}$ and $v_{1}^{2} \nu \theta_{0}=f_{8,2}^{(a)}$. Hence, by the proof of Lemma 4, it suffices to show that $\rho_{1} T^{3}(\omega)\left(e_{1}^{8}\right)=\rho_{1} T^{3}(\omega)\left(e_{2}^{8}\right)=\rho_{1} T^{3}(\omega)\left(e_{3}^{8}\right)=0$ and $\rho_{1} T^{3}(\omega)\left(e_{4}^{8}\right)=2 \beta_{1}\left(a+a b^{r}\right)+\beta_{2} a$ for some integers $\beta_{1}, \beta_{2}$. Part 2 of Lemma 6 implies that $T^{3}(\omega)\left(e_{1}^{8}\right)=T^{3}(\omega)\left(e_{2}^{8}\right)=T^{3}(\omega)\left(e_{3}^{8}\right)=0$ and

$$
\rho_{1} T^{3}(\omega)\left(e_{4}^{8}\right)=x_{2}\left(-x_{1} t^{4} a * S_{1} a+\frac{r^{2}}{8} y_{2}^{2} t^{2}\left(a+a b^{r}\right)\right) .
$$

Since $a * S_{1} a$ is of the form (6.3), we obtain $\rho_{1} \omega=0$. Multiplying this by $\theta_{1}$, we see that $\left(1+\lambda^{\frac{r}{2}}\right) \omega^{2}=0$, whence $\omega^{2}=0$. Multiplying the last identity by $\theta_{1}$, we obtain $v_{1} \rho_{2} \omega=0$. Since $\rho_{2} \omega \in \mathrm{H}^{10}\left(G, M^{(a)}(0)\right)$, we easily show that $\rho_{2} \omega=\varkappa \nu^{2} \theta_{1}$ for some $\varkappa \in \mathbb{Z}$. Multiplying this by $\theta_{0}$, we see that $\varkappa \equiv 0(\bmod r)$, whence $\rho_{2} \omega=0$. Then $v_{1} \rho_{1} \rho_{2}=\theta_{0} \omega \rho_{2}=0$, and $v_{1} \rho_{2}^{2}=\theta_{1} \omega \rho_{2}=0$, implying that $\rho_{1} \rho_{2}=\left(\varkappa_{1}+\varkappa_{1}^{\prime} \lambda^{\frac{r}{2}}\right) \nu^{2}$ and $\rho_{2}^{2}=\left(\varkappa_{2}+\varkappa_{2}^{\prime} \lambda^{\frac{r}{2}}\right) v_{2} \nu^{2}$ for some $\varkappa_{1}, \varkappa_{1}^{\prime}, \varkappa_{2}, \varkappa_{2}^{\prime} \in \mathbb{Z}$. To prove that $\varkappa_{1}=\varkappa_{1}^{\prime}=\varkappa_{2}=\varkappa_{2}^{\prime}=$ 0 , we can argue as in the proof of $\varkappa_{3}=\varkappa_{3}^{\prime}=0$ in (6.4). Consequently, $\rho_{1} \rho_{2}=\rho_{2}^{2}=0$.

The relations for $\varphi_{0}^{2}$ and $\varphi_{0} \varphi_{1}$ are proved like those for $\psi_{0}^{2}$ and $\psi_{0} \psi_{1}$. Moreover, using part 5) of Lemma 6, we establish that $\varphi_{1}^{2}$ is represented in $\mathrm{H}^{4}(G, \widetilde{R})$ by an element that takes $e_{1}^{4}$ to 0 and $e_{5}^{4}$ to $\left(r^{2}+2 r+2\right) b^{2}+(2 r+2) b^{r+2}$. From (3.6) , it follows that, as an element of $\mathrm{H}^{4}(G, \mathbb{Z}(0)) \oplus \mathrm{H}^{4}\left(G, \mathbb{Z}\left(\frac{r}{2}\right)\right), \varphi_{1}^{2}$ is presented in the form $\left(2 f_{4,2}, 2 f_{4,2}\right)$, whence $\varphi_{1}^{2}=2\left(\lambda+\lambda^{\frac{r+2}{2}}\right) \nu=\varphi_{0}^{2} \nu$.

Since $\varphi_{0} T^{0}\left(v_{1}\right)\left(e_{3}^{2}\right)=\varphi_{1} T^{2}\left(v_{1}\right)\left(e_{5}^{4}\right)=0$ (see part 1 of Lemma 5) and $\varphi_{0} T^{0}\left(v_{2}\right)\left(e_{3}^{2}\right)=$ $2\left(b+b^{t}\right), \varphi_{1} T^{2}\left(v_{2}\right)\left(e_{5}^{4}\right)=2\left(b+b^{t}\right)+2 r b$ (see part 1 of Lemma 6), we obtain $\varphi_{0} v_{1}=$ $\varphi_{1} v_{1}=0, \varphi_{0} v_{2}=2 \varphi_{1}, \varphi_{1} v_{2}=2 \varphi_{0} \nu$. The identities $\varphi_{0} \omega=\varphi_{1} \omega=0$ are fulfilled because $\mathrm{H}^{2 n+1}\left(G, M^{(b)}\right)=0$ for any $n$. Moreover, $\lambda^{\frac{r}{2}} \varphi_{0}=\varphi_{0}$, and $\mu\left(\lambda^{\frac{r}{2}} \otimes \mathrm{id}\right) T^{0}\left(\varphi_{1}\right)=$ $(r+1) f_{2,1}^{(b)}-\frac{r+2}{2} g_{2,1}^{(b)}=(r+1) \varphi_{1}$.

Next, it is immediately verified that $\psi_{0} \theta_{0}=2 \varphi_{0}$ and $\mu\left(\psi_{0} \otimes \mathrm{id}\right) T^{0}\left(\theta_{1}\right)\left(e_{3}^{2}\right)=2\left(b+b^{t}\right)=$ $\mu\left(\theta_{0} \otimes \mathrm{id}\right) T^{0}\left(\psi_{1}\right)\left(e_{3}^{2}\right)$. Moreover, part 4 of Lemma 6 shows that $\mu\left(\psi_{1} \otimes \mathrm{id}\right) T^{2}\left(\theta_{1}\right)\left(e_{5}^{4}\right)=$ $2\left(b+b^{t}\right)$. The identities obtained above (and the calculation of the groups $\mathrm{H}^{2 m}\left(G, M^{(b)}\right)$, see the proof of Lemma 3) show that $\psi_{0} \theta_{1}=\psi_{1} \theta_{0}=2 \varphi_{1}$ and $\psi_{1} \theta_{1}=2 \varphi_{0} \nu$. The relations $\psi_{0} \rho_{1}=\psi_{0} \rho_{2}=\psi_{1} \rho_{1}=\psi_{1} \rho_{2}=0$ are valid because $\mathrm{H}^{2 n+1}\left(G, M^{(b)}\right)=0$ for any $n$.

Now, direct calculations show that $\psi_{0} \varphi_{0}=2 \lambda \theta_{0}$ and that in $\mathrm{H}^{2}\left(G, M^{(a)}(1)\right)$ we have $\psi_{0} \varphi_{1}=-\frac{r+2}{2} f_{2,1}^{(a)}=2 g_{2,0}^{(a)}, \varphi_{0} \psi_{1}=-f_{2,1}^{(a)}=2 g_{2,0}^{(a)}$. Consequently, $\varphi_{0} \psi_{1}=\varphi_{1} \psi_{0}=2 \theta_{1} \lambda$. Since $v_{1} \varphi_{1} \psi_{1}=0$ and $\varphi_{1} \psi_{1} \in \mathrm{H}^{4}\left(G, M^{(a)}(1)\right)$, we have $\varphi_{1} \psi_{1}=\varkappa \theta_{0} \nu \lambda$ for some $\varkappa \in \mathbb{Z}$. Multiplying the last identity by $\theta_{0}$ and using the identities proved above, we see that $\varkappa \equiv 2(\bmod r)$, whence, $\varphi_{1} \psi_{1}=2 \theta_{0} \nu \lambda$.

Again, direct calculations show that $\theta_{0} \varphi_{0}=2 \psi_{0}$, and that in $\mathrm{H}^{2}\left(G, M^{(a b)}(0)\right)$ we have

$$
\theta_{0} \varphi_{1}=(r+2) f_{2,1}^{(a b)}-\frac{r+2}{2} g_{2,1}^{(a b)}=2 f_{2,1}^{(a b)} \text { and } \varphi_{0} \theta_{1}=2 f_{2,1}^{(a b)}-g_{2,1}^{(a b)}=(r+2) f_{2,1}^{(a b)}
$$


i.e., $\theta_{0} \psi_{1}=2 \varphi_{1}$ and $\theta_{1} \psi_{0}=(2+r) \varphi_{1}$. Using part 4) of Lemma 6, we check that

$$
\begin{aligned}
& \mu\left(\varphi_{1} \otimes \mathrm{id}\right) T^{2}\left(\theta_{1}\right)\left(e_{3}^{4}\right)=\frac{r^{2}+r+2}{2} x_{1}\left(a b+a b^{t}\right), \\
& \mu\left(\varphi_{1} \otimes \mathrm{id}\right) T^{2}\left(\theta_{1}\right)\left(e_{4}^{4}\right)=-\frac{r^{2}+5 r+4}{2}\left(a b+a b^{t}\right)+r a b, \\
& \mu\left(\varphi_{1} \otimes \mathrm{id}\right) T^{2}\left(\theta_{1}\right)\left(e_{5}^{4}\right)=(r+2)\left(a b+a b^{t}\right) .
\end{aligned}
$$

From (3.8) and (3.9), it follows that in $\mathrm{H}^{4}\left(G, M^{(a b)}(0)\right)$ we have

$$
\varphi_{1} \theta_{1}=\frac{r^{2}+r+2}{2} f_{4,1}^{(a b)}-\frac{r^{2}+5 r+4}{2} g_{4,2}^{(a b)}+(r+2) f_{4,2}^{(a b)}=2 f_{4,2}^{(a b)}=2 \psi_{0} \nu .
$$

Finally, since $\mathrm{H}^{2 n+1}\left(G, M^{(a b)}\right)=0$ for any $n$, we have

$$
\varphi_{0} \rho_{1}=\varphi_{0} \rho_{2}=\varphi_{1} \rho_{1}=\varphi_{1} \rho_{2}=0 .
$$

Corollary 5. The algebra $\mathrm{HH}^{*}\left(\mathbb{Z}\left[M_{2} \ell\right]\right)$ is commutative.

Proof. This follows from the fact that the product of any two generators of odd degree (namely, they are exactly $\omega, \rho_{1}$, and $\rho_{2}$ ) is equal to 0 .

\section{$\S 7$. Description of the Hochschild COHOMOlOGy Ring}

In this section, we describe the Hochschild cohomology algebra of the group $G$ in terms of generators and defining relations. Put $\mathcal{X}=\left\{\lambda, v_{1}, v_{2}, \nu, \omega, \varphi_{0}, \varphi_{1}, \theta_{0}, \theta_{1}, \rho_{1}, \rho_{2}, \psi_{0}, \psi_{1}\right\}$; the elements of this set were defined in $\S 4$. Then we consider the new set $\widetilde{\mathcal{X}}=\{\widetilde{x} \mid x \in \mathcal{X}\}$, which is in a one-to-one correspondence with $\mathcal{X}$. On the algebra $\mathbb{Z}[\tilde{\mathcal{X}}]$, we introduce a grading such that $\operatorname{deg} \tilde{x}=\operatorname{deg} x$ for any $x \in \mathcal{X}$.

We consider the algebra $\mathcal{H}=\mathbb{Z}[\tilde{\mathcal{X}}] / \mathcal{I}$, where $\mathcal{I}$ is the homogeneous ideal generated by the following elements:

$$
\begin{aligned}
& 2 \widetilde{v}_{1}, r \widetilde{v}_{2}, 2 r \widetilde{\nu}, 2 \widetilde{\omega}, 2 r \widetilde{\varphi}_{1}, r \widetilde{\theta}_{0} \widetilde{\nu}, r \widetilde{\theta}_{1}, 2 \widetilde{\rho}_{1}, 2 \widetilde{\rho}_{2}, 2 r \widetilde{\psi}_{1}, \\
& \widetilde{v}_{1} \widetilde{v}_{2}, \widetilde{v}_{2}^{2}-4 \widetilde{\nu}, \widetilde{v}_{2} \widetilde{\omega}, \widetilde{\omega}^{2}, \tilde{\lambda}^{r}-1 \text {, } \\
& \widetilde{\psi}_{0}^{2}-2\left(\widetilde{\lambda}+\widetilde{\lambda}^{\frac{r+2}{2}}\right), \widetilde{\psi}_{0} \widetilde{\psi}_{1}-\left(\widetilde{\lambda}+\tilde{\lambda}^{\frac{r+2}{2}}\right) \widetilde{v}_{2}, \widetilde{\psi}_{1}^{2}-\widetilde{\psi}_{0}^{2} \widetilde{\nu} \\
& \widetilde{\psi}_{0} \widetilde{v}_{1}-r \widetilde{\psi}_{1}, \widetilde{\psi}_{1} \widetilde{v}_{1}-r \widetilde{\psi}_{0} \widetilde{\nu}, \widetilde{\psi}_{0} \widetilde{v}_{2}-(2+r) \tilde{\psi}_{1}, \widetilde{\psi}_{1} \widetilde{v}_{2}-(2+r) \tilde{\psi}_{0} \widetilde{\nu}, \widetilde{\psi} 0 \tilde{\omega}, \widetilde{\psi}_{1} \widetilde{\omega}, \\
& \widetilde{\psi}_{0} \tilde{\lambda}^{\frac{r}{2}}-\widetilde{\psi}_{0}, \widetilde{\psi}_{1} \widetilde{\lambda}^{\frac{r}{2}}-(1+r) \widetilde{\psi}_{1} \\
& \widetilde{\theta}_{0}^{2}-2\left(1+\tilde{\lambda}^{\frac{r}{2}}\right), \widetilde{\theta}_{0} \widetilde{\theta}_{1}-\left(1+\widetilde{\lambda}^{\frac{r}{2}}\right)\left(\widetilde{v}_{1}+\widetilde{v}_{2}\right), \widetilde{\theta}_{1}^{2}-\widetilde{v}_{1}^{2}-\widetilde{\theta}_{0}^{2} \widetilde{\nu}, \widetilde{\theta}_{0} \widetilde{\rho}_{1}, \\
& \widetilde{\theta}_{1} \widetilde{\rho}_{1}-\widetilde{\theta}_{0} \widetilde{\rho}_{2}, \widetilde{\theta}_{0} \tilde{\rho}_{2}-\left(1+\widetilde{\lambda}^{\frac{r}{2}}\right) \widetilde{\omega}, \widetilde{\theta}_{1} \widetilde{\rho}_{2}-\widetilde{v}_{1} \widetilde{\omega}, \widetilde{\rho}_{1}^{2}, \widetilde{\rho}_{1} \tilde{\rho}_{2}, \widetilde{\rho}_{2}^{2}, \\
& \widetilde{\theta}_{0} \widetilde{v}_{2}-2 \widetilde{\theta}_{1}, \widetilde{\theta}_{1} \widetilde{v}_{2}-2 \widetilde{\theta}_{0} \widetilde{\nu}, \widetilde{\theta}_{0} \widetilde{\omega}-\widetilde{\rho}_{1} \widetilde{v}_{1}, \widetilde{\theta}_{1} \widetilde{\omega}-\widetilde{\rho}_{2} \widetilde{v}_{1}, \widetilde{\rho}_{1} \widetilde{v}_{2}, \widetilde{\rho}_{2} \widetilde{v}_{2}, \tilde{\rho}_{1} \widetilde{\omega}, \widetilde{\rho}_{2} \widetilde{\omega}, \\
& \widetilde{\theta}_{0} \widetilde{\lambda}^{\frac{r}{2}}-\widetilde{\theta}_{0}, \widetilde{\theta}_{1} \widetilde{\lambda}^{\frac{r}{2}}-\widetilde{\theta}_{1}-\widetilde{\theta}_{0} \widetilde{v}_{1}, \widetilde{\rho}_{1} \tilde{\lambda}^{\frac{r}{2}}-\widetilde{\rho}_{1}, \widetilde{\rho}_{2} \widetilde{\lambda}^{\frac{r}{2}}-\widetilde{\rho}_{2}-\widetilde{\rho}_{1} \widetilde{v}_{1}, \\
& \widetilde{\varphi}_{0}^{2}-2\left(\tilde{\lambda}+\tilde{\lambda}^{\frac{r+2}{2}}\right), \widetilde{\varphi}_{0} \widetilde{\varphi}_{1}-\left(1+\frac{r}{2}\right)\left(\widetilde{\lambda}+\tilde{\lambda}^{\frac{r+2}{2}}\right) \widetilde{v}_{2}, \widetilde{\varphi}_{1}^{2}-\widetilde{\varphi}_{0}^{2} \widetilde{\nu}, \\
& \widetilde{\varphi}_{0} \widetilde{v}_{1}, \widetilde{\varphi}_{1} \widetilde{v}_{1}, \widetilde{\varphi}_{0} \widetilde{\omega}, \widetilde{\varphi}_{1} \widetilde{\omega}, \widetilde{\varphi}_{0} \widetilde{v}_{2}-2 \widetilde{\varphi}_{1}, \widetilde{\varphi}_{1} \widetilde{v}_{2}-2 \widetilde{\varphi}_{0} \widetilde{\nu}, \widetilde{\varphi}_{0} \widetilde{\lambda}^{\frac{r}{2}}-\widetilde{\varphi}_{0}, \widetilde{\varphi}_{1} \tilde{\lambda}^{\frac{r}{2}}-(1+r) \tilde{\varphi}_{1}, \\
& \widetilde{\psi}_{0} \widetilde{\theta}_{0}-2 \widetilde{\varphi}_{0}, \widetilde{\psi}_{0} \widetilde{\theta}_{1}-2 \widetilde{\varphi}_{1}, \widetilde{\psi}_{1} \widetilde{\theta}_{0}-2 \widetilde{\varphi}_{1}, \widetilde{\psi}_{1} \widetilde{\theta}_{1}-2 \widetilde{\varphi}_{0} \widetilde{\nu}, \widetilde{\psi}_{0} \widetilde{\rho}_{1}, \widetilde{\psi}_{0} \widetilde{\rho}_{2}, \widetilde{\psi}_{1} \tilde{\rho}_{1}, \widetilde{\psi}_{1} \tilde{\rho}_{2}, \\
& \widetilde{\varphi}_{0} \widetilde{\psi}_{0}-2 \widetilde{\theta}_{0} \tilde{\lambda}, \widetilde{\varphi}_{0} \widetilde{\psi}_{1}-2 \widetilde{\theta}_{1} \tilde{\lambda}, \widetilde{\varphi}_{1} \widetilde{\psi}_{0}-2 \widetilde{\theta}_{1} \tilde{\lambda}, \widetilde{\varphi}_{1} \widetilde{\psi}_{1}-2 \widetilde{\theta}_{0} \tilde{\nu} \tilde{\lambda}
\end{aligned}
$$

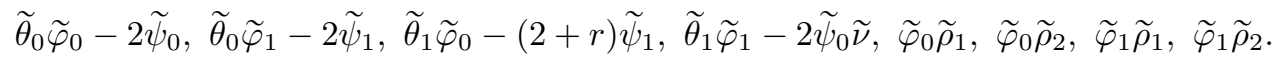

Theorem 2. There is an isomorphism $\mathrm{HH}^{*}(R) \simeq \mathcal{H}$ of graded $\mathbb{Z}$-algebras. 
Proof. Since $\mathrm{HH}^{*}(R) \simeq \mathrm{H}^{*}(G, \widetilde{R})$, it suffices to prove that there exists an isomorphism of graded $\mathbb{Z}$-algebras $\mathcal{H} \simeq \mathrm{H}^{*}(G, \widetilde{R})$. From Propositions 2 and 3 , it follows that there exists a surjective homomorphism $\pi: \mathcal{H} \rightarrow \mathrm{H}^{*}(G, \widetilde{R})$ of graded $\mathbb{Z}$-algebras, specifically, it is defined on the classes of elements of the set $\widetilde{\mathcal{X}}$ by the formula $\pi(\widetilde{x}+\mathcal{I})=x$. For simplicity, in what follows we write $\widetilde{x}$ instead of $\widetilde{x}+\mathcal{I}$. Let $\mathcal{H}=\bigoplus_{n \geq 0} \mathcal{H}_{n}$ be a decomposition of $\mathcal{H}$ into homogeneous direct summands. Now it suffices to establish that $\pi_{n}=\left.\pi\right|_{\mathcal{H}_{n}}$ is an isomorphism for any $n \geq 0$.

Using the proof of Proposition 2, we conclude easily that multiplication by $\nu$ induces an embedding of $\mathrm{H}^{n}(G, \widetilde{R})$ into $\mathrm{H}^{n+4}(G, \widetilde{R})$ for $n>0$. By definition, we have

$$
\mathcal{H}_{n}={ }_{\mathbb{Z}}\left\langle\widetilde{x}_{1} \ldots \widetilde{x}_{p} \mid p \geq 0, x_{i} \in \mathcal{X}(1 \leq i \leq p), \sum_{i=1}^{p} \operatorname{deg} x_{i}=n\right\rangle .
$$

Observe that $\mathcal{X}^{\prime}=\left\{\omega, \rho_{1}, \rho_{2}\right\}$ is the set of all elements of odd degree in $\mathcal{X}$, and moreover, $\widetilde{\omega}^{2}, \widetilde{\rho}_{1}^{2}, \widetilde{\rho}_{2}^{2}, \widetilde{\rho}_{1} \widetilde{\omega}, \widetilde{\rho}_{2} \widetilde{\omega}, \widetilde{\rho}_{1} \widetilde{\rho}_{2} \in \mathcal{I}$. Consequently,

$$
\begin{gathered}
\mathcal{H}_{2 k}={ }_{\mathbb{Z}}\left\langle\widetilde{x}_{1} \ldots \widetilde{x}_{p} \mid p \geq 0, x_{i} \in \mathcal{X} \backslash \mathcal{X}^{\prime}(1 \leq i \leq p), \sum_{i=1}^{p} \operatorname{deg} x_{i}=2 k\right\rangle, \\
\mathcal{H}_{2 k+1}={ }_{\mathbb{Z}}\left\langle\widetilde{x}_{1} \ldots \widetilde{x}_{p} \mid p \geq 1, x_{i} \in \mathcal{X} \backslash \mathcal{X}^{\prime}(1 \leq i \leq p-1), x_{p} \in \mathcal{X}^{\prime}, \sum_{i=1}^{p} \operatorname{deg} x_{i}=2 k+1\right\rangle .
\end{gathered}
$$

First, we check that $\pi_{2 k}$ is an isomorphism. We prove this by induction on $k$. Analyzing the form of the generators of the ideal $\mathcal{I}$, we easily show that $\mathcal{H}_{0}$ consists of the $\mathbb{Z}$-linear combinations of elements of the set $\left\{\widetilde{\lambda}^{i}\right\}_{0 \leq i \leq r-1} \cup\left\{\widetilde{\varphi}_{0} \widetilde{\lambda}^{i}, \widetilde{\theta}_{0} \widetilde{\lambda}^{i}, \widetilde{\psi}_{0} \widetilde{\lambda}^{i}\right\}_{0 \leq i \leq \frac{r-2}{2}}$, $\mathcal{H}_{2}$ consists of the $\mathbb{Z}$-linear combinations of elements of the set $\left\{\widetilde{v}_{1} \tilde{\lambda}^{i}, \widetilde{v}_{2} \tilde{\lambda}^{i}\right\}_{0 \leq i \leq r-1} \cup$ $\left\{\widetilde{\varphi}_{1} \tilde{\lambda}^{i}, \widetilde{\theta}_{0} \widetilde{v}_{1} \tilde{\lambda}^{i}, \widetilde{\theta}_{1} \tilde{\lambda}^{i}, \widetilde{\psi}_{1} \tilde{\lambda}^{i}\right\}_{0 \leq i \leq \frac{r-2}{2}}$, and $\mathcal{H}_{4}$ consists of the $\mathbb{Z}$-linear combinations of elements of the set

$$
\left\{\widetilde{v}_{1}^{2} \widetilde{\lambda}^{i}, \widetilde{\nu} \widetilde{\lambda}^{i}\right\}_{0 \leq i \leq r-1} \cup\left\{\widetilde{\varphi}_{0} \widetilde{\nu} \widetilde{\lambda}^{i}, \widetilde{\theta}_{0} \widetilde{v}_{1}^{2} \widetilde{\lambda}^{i}, \widetilde{\theta}_{1} \widetilde{v}_{1} \tilde{\lambda}^{i}, \widetilde{\theta}_{0} \widetilde{\nu} \widetilde{\lambda}^{i}, \widetilde{\psi}_{0} \widetilde{\nu} \widetilde{\lambda}^{i}\right\}_{0 \leq i \leq \frac{r-2}{2}}
$$

Looking at the list of the generators of the ideal $\mathcal{I}$, we see that $\pi_{0}, \pi_{2}$, and $\pi_{4}$ are isomorphisms. Let $k \geq 3$, and suppose that $\pi_{2 j}$ is an isomorphism for any $0 \leq j \leq k-1$. We shall prove that $\pi_{2 k}$ is also an isomorphism. First, we prove that $\mathcal{H}_{2 k}=\widetilde{v}_{1} \mathcal{H}_{2 k-2}+$ $\widetilde{\nu} \mathcal{H}_{2 k-4}$, i.e., any element of the form $\widetilde{x}_{1} \ldots \widetilde{x}_{p}$, where $p \geq 0, x_{i} \in \mathcal{X} \backslash \mathcal{X}^{\prime}(1 \leq i \leq p)$, and $\sum_{i=1}^{p} \operatorname{deg} x_{i}=2 k$, can be presented in $\mathcal{H}$ in the form $\widetilde{v}_{1} X_{2 k-2}+\widetilde{\nu} X_{2 k-4}$ with $X_{2 k-2} \in \mathcal{H}_{2 k-2}, X_{2 k-4} \in \mathcal{H}_{2 k-4}$. The condition $\sum_{i=1}^{p} \operatorname{deg} x_{i}=2 k$ implies that either $x_{i} \in\left\{v_{1}, \nu\right\}$ for some $1 \leq i \leq p$, or $x_{i}, x_{j} \in\left\{v_{2}, \varphi_{1}, \theta_{1}, \psi_{1}\right\}$ for some $1 \leq i<j \leq p$. In the former case, the claim in question is evident, and in the latter case this claim follows from the fact that the product of any two elements of the set $\left\{\widetilde{v}_{2}, \widetilde{\varphi}_{1}, \widetilde{\theta}_{1}, \widetilde{\psi}_{1}\right\}$ can be presented in the required form. Now we have

$$
\begin{aligned}
\mathcal{H}_{2 k} & =\widetilde{v}_{1}^{k-1} \mathcal{H}_{2}+\widetilde{\nu} \mathcal{H}_{2 k-4} \\
& =\widetilde{v}_{1}^{k} \mathcal{H}_{0}+\widetilde{v}_{1}^{k-1} \widetilde{v}_{2} \mathcal{H}_{0}+\widetilde{v}_{1}^{k-1} \widetilde{\varphi}_{1} \mathcal{H}_{0}+\widetilde{v}_{1}^{k-1} \widetilde{\theta}_{1} \mathcal{H}_{0}+\widetilde{v}_{1}^{k-1} \widetilde{\psi}_{1} \mathcal{H}_{0}+\widetilde{\nu} \mathcal{H}_{2 k-4} \\
& =\widetilde{v}_{1}^{k} \mathcal{H}_{0}+\widetilde{v}_{1}^{k-1} \widetilde{\theta}_{1} \mathcal{H}_{0}+\widetilde{\nu} \mathcal{H}_{2 k-4} .
\end{aligned}
$$

Since

$$
\begin{aligned}
\widetilde{v}_{1}^{2} \mathcal{H}_{0} & ={ }_{\mathbb{Z}}\left\langle\left\{\widetilde{v}_{1}^{2} \widetilde{\lambda}^{i}\right\}_{0 \leq i \leq r-1} \cup\left\{\widetilde{v}_{1}^{2} \widetilde{\varphi}_{0} \widetilde{\lambda}^{i}, \widetilde{v}_{1}^{2} \widetilde{\theta}_{0} \widetilde{\lambda}^{i}, \widetilde{v}_{1}^{2} \widetilde{\psi}_{0} \widetilde{\lambda}^{i}\right\}_{0 \leq i \leq \frac{r-2}{2}}\right\rangle \\
& ={ }_{\mathbb{Z}}\left\langle\left\{\widetilde{v}_{1}^{2} \widetilde{\lambda}^{i}\right\}_{0 \leq i \leq r-1} \cup\left\{\widetilde{v}_{1}^{2} \widetilde{\theta}_{0} \widetilde{\lambda}^{i}\right\}_{0 \leq i \leq \frac{r-2}{2}}\right\rangle,
\end{aligned}
$$




$$
\begin{aligned}
& \widetilde{v}_{1} \tilde{\theta}_{1} \mathcal{H}_{0}=\mathbb{Z}\left\langle\left\{\widetilde{v}_{1} \tilde{\theta}_{1} \tilde{\lambda}^{i}\right\}_{0 \leq i \leq r-1} \cup\left\{\widetilde{v}_{1} \tilde{\theta}_{1} \widetilde{\varphi}_{0} \tilde{\lambda}^{i}, \widetilde{v}_{1} \tilde{\theta}_{1} \tilde{\theta}_{0} \tilde{\lambda}^{i}, \widetilde{v}_{1} \tilde{\theta}_{1} \widetilde{\psi}_{0} \tilde{\lambda}^{i}\right\}_{0 \leq i \leq \frac{r-2}{2}}\right\rangle \\
& ={ }_{\mathbb{Z}}\left\langle\left\{\widetilde{v}_{1} \tilde{\theta}_{1} \tilde{\lambda}^{i}\right\}_{0 \leq i \leq r-1} \cup\left\{2 \widetilde{v}_{1} \widetilde{\psi}_{1} \tilde{\lambda}^{i}, \widetilde{v}_{1}\left(1+\tilde{\lambda}^{\frac{r}{2}}\right)\left(v_{1}+v_{2}\right) \tilde{\lambda}^{i},(2+r) \widetilde{v}_{1} \widetilde{\varphi}_{1} \tilde{\lambda}^{i}\right\}_{0 \leq i \leq \frac{r-2}{2}}\right\rangle \\
& =\mathbb{Z}\left\langle\left\{\widetilde{v}_{1} \widetilde{\theta}_{1} \tilde{\lambda}^{i}\right\}_{0 \leq i \leq r-1} \cup\left\{\widetilde{v}_{1}^{2}\left(\tilde{\lambda}^{i}+\tilde{\lambda}^{i+\frac{r}{2}}\right)\right\}_{0 \leq i \leq \frac{r-2}{2}}\right\rangle,
\end{aligned}
$$

and

we obtain

$$
\mathbb{Z}\left\langle\left\{\widetilde{v}_{1} \widetilde{\theta}_{1} \tilde{\lambda}^{i}\right\}_{0 \leq i \leq r-1}\right\rangle=\mathbb{Z}\left\langle\left\{\widetilde{v}_{1} \tilde{\theta}_{1} \tilde{\lambda}^{i}, \widetilde{v}_{1} \tilde{\theta}_{1} \tilde{\lambda}^{i}+\widetilde{v}_{1}^{2} \widetilde{\theta}_{0} \tilde{\lambda}^{i}\right\}_{0 \leq i \leq \frac{r-2}{2}}\right\rangle,
$$

$$
\mathcal{H}_{2 k}={ }_{\mathbb{Z}}\left\langle\left\{\widetilde{v}_{1}^{k} \tilde{\lambda}^{i}\right\}_{0 \leq i \leq r-1} \cup\left\{\widetilde{v}_{1}^{k} \widetilde{\theta}_{0} \tilde{\lambda}^{i}, \widetilde{v}_{1}^{k-1} \widetilde{\theta}_{1} \tilde{\lambda}^{i}\right\}_{0 \leq i \leq \frac{r-2}{2}}\right\rangle+\widetilde{\nu} \mathcal{H}_{2 k-4} .
$$

Since $2 v_{1}=0$ and $\left|\mathcal{H}_{2 k-4}\right|=\left|\mathrm{H}^{2 k-4}(G, \widetilde{R})\right|$ by the inductive hypothesis, we conclude that

$$
\left|\mathcal{H}_{2 k}\right| \leq 2^{2 r}\left|\mathcal{H}_{2 k-4}\right|=2^{2 r}\left|\mathrm{H}^{2 k-4}(G, \widetilde{R})\right|=\left|\mathrm{H}^{2 k}(G, \widetilde{R})\right| \text { (see Theorem 1). }
$$

Since the map $\pi_{2 k}$ is surjective, it must be an isomorphism.

Now we prove that $\pi_{2 k+1}$ is an isomorphism. Using the form of the generators of the ideal $\mathcal{I}$, we can rewrite (7.2) as follows:

$$
\mathcal{H}_{2 k+1}={ }_{\mathbb{Z}}\left\langle\widetilde{x}_{1} \ldots \tilde{x}_{p} \mid p \geq 1, x_{i} \in\left\{v_{1}, \nu, \lambda\right\}(1 \leq i \leq p-1), x_{p} \in \mathcal{X}^{\prime}, \sum_{i=1}^{p} \operatorname{deg} x_{i}=n\right\rangle .
$$

We use induction on $k$ to check that $\left|\mathcal{H}_{2 k+1}\right| \leq\left|\mathrm{H}^{2 k+1}(G, \widetilde{R})\right|$. First, observe that $\mathcal{H}_{1}=0=\mathrm{H}^{1}(G, \widetilde{R})$ and $\left|\mathcal{H}_{3}\right|=\left|\mathbb{Z}\left\langle\left\{\widetilde{\rho}_{1} \widetilde{\lambda}^{i}\right\}_{0 \leq i \leq \frac{r-2}{2}}\right\rangle\right| \leq 2^{\frac{r}{2}}=\left|\mathrm{H}^{3}(G, \widetilde{R})\right|$. Next, we assume that $k \geq 2$ and $\left|\mathcal{H}_{2 j+1}\right| \leq\left|\mathrm{H}^{2 j+1}(G, \widetilde{R})\right|$ for any $0 \leq j \leq k-1$. With the help of (7.3), we verify that

$\mathcal{H}_{2 k+1}=\widetilde{\nu} \mathcal{H}_{2 k-3}+{ }_{\mathbb{Z}}\left\langle\left\{\widetilde{\rho}_{1} \widetilde{v}_{1}^{k-1} \widetilde{\lambda}^{i}\right\}_{0 \leq i \leq \frac{r-2}{2}} \cup\left\{\widetilde{\rho}_{2} \widetilde{v}_{1}^{k-2} \widetilde{\lambda}^{i}\right\}_{0 \leq i \leq \frac{r-2}{2}} \cup\left\{\widetilde{\omega} \widetilde{v}_{1}^{k-2} \widetilde{\lambda}^{i}\right\}_{0 \leq i \leq r-1}\right\rangle$.

Then $\left|\mathcal{H}_{2 k+1}\right| \leq 2^{2 r}\left|\mathcal{H}_{2 k-3}\right| \leq 2^{2 r}\left|\mathrm{H}^{2 k-3}(G, \widetilde{R})\right|=\left|\mathrm{H}^{2 k+1}(G, \widetilde{R})\right|$ (see Theorem 10). Hence, we have $\left|\mathcal{H}_{2 k+1}\right| \leq\left|\mathrm{H}^{2 k+1}(G, \widetilde{R})\right|$ for any $k \geq 0$, and the surjectivity of $\pi$ implies that $\pi_{2 k+1}$ is an isomorphism.

Corollary 6. The graded algebra $\mathrm{H}^{*}(G, \mathbb{Z})$ is isomorphic to $\mathbb{Z}\left[\widetilde{v}_{1}, \widetilde{v}_{2}, \widetilde{\nu}, \widetilde{\omega}\right] / I$, where $\operatorname{deg} \widetilde{v}_{1}=\operatorname{deg} \widetilde{v}_{2}=2, \operatorname{deg} \widetilde{\nu}=4, \operatorname{deg} \widetilde{\omega}=5$, and $I$ is the ideal generated by the elements $2 \widetilde{v}_{1}, r \widetilde{v}_{2}, 2 r \widetilde{\nu}, 2 \widetilde{\omega}, \widetilde{v}_{1} \widetilde{v}_{2}, \widetilde{v}_{2}^{2}-4 \widetilde{\nu}, \widetilde{v}_{2} \widetilde{\omega}$, and $\widetilde{\omega}^{2}$.

Proof. This follows from Corollary 4 and the proof of Theorem 2.

\section{REFERENCES}

[1] A. I. Generalov, The Hochschild cohomology of the integer group ring of a dihedral group. I, The even case, Algebra i Analiz 19 (2007), no. 5, 70-123; English transl., St. Petersburg Math. J. 19 (2008), no. 5, 723-763. MR2381942 (2009e:16015)

[2] _ Hochschild cohomology for the integer group ring of the semidihedral group, Zap. Nauchn. Sem. S.-Petersburg. Otdel. Mat. Inst. Steklov. (POMI) 388 (2011), 119-151; English transl., J. Math. Sci. (N.Y.) 183 (2012), no. 5, 640-657. MR2822518(2012e:16024)

[3] T. Hayami, Hochschild cohomology ring of the integral group ring of the generalized quaternion group, SUT J. Math. 38 (2002), no. 1, 83-126. MR1919950(2003f:16014)

[4] - On Hochschild cohomology ring of the integral group ring of the quaternion group, Tsukuba J. Math. 29 (2005), no. 2, 363-387. MR2177018 (2006j:16016)

[5] - Hochschild cohomology ring of the integral group ring of dihedral groups, Tsukuba J. Math. 31 (2007), no. 1, 99-127. MR2337122 (2008d:16016)

[6] _ Hochschild cohomology ring of the integral group ring of the semidehidral 2-group, Algebra Colloq. 18 (2011), no. 2, 241-258. MR2785780 (2012c:16031) 
[7] S. F. Siegel and S. J. Witherspoon, The Hochschild cohomology ring of a group algebra, Proc. London Math. Soc.(3) 79 (1999), no. 1, 131-157. MR1687539 (2000b:16016)

[8] C. T. C. Wall, Resolutions for extensions of groups, Proc. Cambridge Philos. Soc. 57 (1961), 251255. MR $0178046(31: 2304)$

[9] A. I. Generalov and S. O. Ivanov, Bimodule resolution of a group algebra, Zap. Nauchn. Sem. S.Peterburg. Otdel. Mat. Inst. Steklov. (POMI) 365 (2009), 143-150; English transl., J. Math. Sci. (N.Y.) 161 (2009), no. 4, 537-541. MR2749138(2012b:16016)

[10] I. J. Leary, The cohomology of certain groups, PhD Thesis, Cambridge, 1992.

[11] C. B. Thomas, Chern classes and metacyclic p-groups, Mathematika 18 (1971), 196-200. MR0299699 (45:8747)

[12] D. J. Benson, Representations and cohomology. II, Cohomology of groups and modules, Cambridge Stud. Adv. Math. vol. 30, Cambridge Univ. Press, Cambridge, 1998. MR.1634407 (99f:20001b)

[13] S. Eilenberg and S. MacLane, Cohomology theory in abstract groups. I, Ann. of Math. (2) 48 (1947), 51-78. MR0019092 (8:367f)

[14] S. MacLane, Homology, Grundlehren Math. Wiss., Bd. 114, Springer-Verlag, Berlin, 1963. $\operatorname{MR} 0156879(28: 122)$

[15] K. Brown, Cohomology of groups, Grad. Texts in Math., vol. 87, Springer, Berlin, 1982. MR672956 (83k:20002)

Research Group, Era7 Bioinformatics, Plaza Campo Verde 3 Atico, Granada 18001, Spain

E-mail address: aalekhin@ohnosequences.com

Department of Mathematics and Mechanics, St. Petersburg State University, UniversitetSkit Pr., 28, Staryi Peterhof, St. Petersburg 198504, Russia

E-mail address: wolf86_666@list.ru

Department of Mathematics and Mechanics, St. Petersburg State University, UniversitetSkit Pr., 28, Staryi Peterhof, St. Petersburg 198504, Russia

E-mail address: ageneralov@gmail.com

Received 10/MAR/2013

Translated by A. I. GENERALOV 